\title{
Determination of phenolic compositions and quality characteristic of some local Turkish table grape varieties cultivated in Egirdir/lsparta
}

\author{
Seckin Gargin ${ }^{1}$ and Ahmet Altindisli ${ }^{2}$ \\ ${ }^{1}$ Egirdir Fruit Research Station Egirdir/Isparta, Turkey \\ ${ }^{2}$ Aegean University, Faculty of Agriculture, Department of Horticulture, İzmir, Turkey
}

\begin{abstract}
This study was carried out to determine the phenolic substances and antioxidant activities of 11 local grape cultivars (Burdur Dimriti, Siyah Gemre, Ak Gemre, Antep Büzgülü, Şam Büzgülü, Kuş Yüregi, Ak Dimrit, Marzımat, Senirkent Dimriti, Devegözü, Tilki Kuyrugu) grown in Egirdir/Isparta province of Turkey by the means of High Performance Liquid Chromatography (HPLC) and also were evaluated for their yield and quality characterics. Burdur Dimriti, Antep Büzgülü, Şam Büzgülü cultivars were evaluated good for yield and table consumption. Ak Gemre, Marzımat, Devegözü, Ak Dimrit, Senirkent Dimriti and Kuş Yüregi varieties were evaluated good for must production. Gallic acid, cafeic acid, p-cumaric acid, ferulic acid, catechin, epicatechin, epicatechin gallate, epigallocatechin, epigallocatechin-3-gallate, kaemferol, myricetin, rutin hydrate, resveratrol, quercetin were determined in berry samples of 11 cultivars. Phenolic substances varied between 0 and $101.466 \mu \mathrm{g} / \mathrm{ml}$ in frozen berry samples varying to grape variety. Some of the cultivars can be evaluated as a good source of resveratrol, gallic acid, quercetin, and catechin. Antep Büzgülü, Burdur Dimriti, Kuş Yüregi, Senirkent Dimriti, Siyah Gemre were evaluated hopeful phenolic sources for further studies. Further detailed studies will be planned about phenolic and antioxidant compositions of these hopeful local varieties according to research results.
\end{abstract}

\section{Introduction}

Grape, cultivated since ancient times is one of the most significant edible and processing for wine crop due to its beneficial influences on human health and economic significance on a large scale (6) and Turkey is one of the major players in this market: according to FAO records, Turkey placed 6th position in the World in terms of amount of grape production according to the data of 2013.

Grapes composition revealed that phenolic compounds are the most widely distributed component within the plant secondary metabolites. Thousands of phenolic compounds with the different characteristics, amounts, and functions have been identified [10,11]. Phenolic compounds in foods cause variation in various organoleptic qualities like taste, bitterness and sourness; and color properties are also altered with phenolic compounds and anthocyanins. Moreover, attributable to beneficial effects on human health in particular, importance of phenolic compounds has increased with each passing day [12]. Phenolic compounds from plants source are categorized into two groups as "phenolic acids" and "flavanoids" while phenolic acids are further subcategorized into two groups as cinamic (hydroxycinamics) and benzoic (hydroxybenzoic) acids [15].

Phenolic compounds are a subdivision of the phytochemical group and they have attracted passionate interest of both researchers and consumers due to their antioxidants and antimicrobial activities [1]. The antioxidant activities of phenolic compounds are attributed to their free radical scavenging and metal chelating properties, as well as their effects on cell signaling pathways and on gene expression
[2-4]. The mechanism of the antioxidant activity has been mainly influenced by the number of $\mathrm{OH}$ groups and their position on the ring in the molecule, which determines the antioxidant capacity of phenolic compounds [2]. The phenolics in grapes are classified in two groups: the flavonoids and non-flavonoids. In flavonoids group are present flavan3-ols (catechin), flavonols (quercetin) and anthocyanins. The nonflavonoids group contains gallic acid, hydroxycinnamates and stilbenes (resveratrol) [2]. Phenolic compounds were indicated to reduce coronary heart diseases [7], some cancer types and various dermal disorders (Yilmaz and Toledo, 2004). The antioxidant activity of grapes is direct correlated with total phenolic content as it is demonstrated by some papers [5]. Despite the existence of thousands of cultivars of Vitis vinifera in grape producer countries, only a few dozen cultivars account for the vast majority of world-wide production. Numerous local cultivars have regional importance and historically played a significant role in many viticulture regions [6]. In fact local cultivars show great variability in particular given biochemical traits and they can be use locally for wine, raisins, and fresh market table grapes [8]. Therefore it is crucial to determine how much variability can be found in local grape cultivars for the given biochemical traits to use them in breeding activities. This may also help the growers to pick the right cultivar according to their market opportunities. Several variables viz. total soluble sugar, titratable acidity; nitrogen and phenolic compounds balance provide a major contribution in the description of the grape quality [9], which are very significant in case of table grape. Therefore, due to beneficial effects of the grape on human 
health, the researchers have more focused in particular on the identification of biochemical composition and bioactive compounds for grape cultivars grown in distinct geographic regions throughout the World.

Various studies are conducted in the world to determine the phenolic compounds and antioxidant activities of different grape varieties $[13,14]$. Conversely, the Turkey has great biodiversity in grapes especially Isparta province (located in west south Anatolia of Turkey known as Lakes Region) and limited studies are conducted on phenolic substances and antioxidant contents of the grapes cultivars grown in this region. This study was carried out to determine yield and quality characterics and also the phenolic compounds (Gallic acid, cafeic acid, cumaric acid, ferulic acid, catechin, epicatechin, epicatechin gallate, epigallocatechin, epigallocatechin 3 gallate, kaemferol, myricetin, rutin hydrate, resveratrol, quercetin) of the grapes genotypes (Burdur Dimriti, Siyah Gemre, Ak Gemre, Antep Büzgülü, Şam Büzgülü, Kuş Yüregi, Ak Dimrit, Marzımat, Senirkent Dimriti, Devegözü, Tilki Kuyrugu) grown in Eğirdir/Isparta province of the Turkey by the means of HPLC.

\section{Material and method}

This study was carried out in Eğirdir Fruit Research Station farmlands during 2012-2013 vegetation season products. Eğirdir is a district of Isparta. Its altitude is $920 \mathrm{~m}$ high and it represents passing zone climate and ecological conditions (Figs. 2.2, 2.3). Geographical coordinates of Eğirdir district; are $37^{\circ} 50$ ' $41^{\prime \prime}, 38^{\circ} 16^{\prime} 55^{\prime \prime} \mathrm{N}$ latitude, $30^{\circ} 57^{\prime} 43^{\prime \prime}, 30^{\circ} 44^{\prime} 39^{\prime \prime}$ E latitude [16]. Isparta has ideal ecological conditions for viticulture in the Lakes Region area. Average rainfall is between 445 and $764 \mathrm{~mm}$. Temperature varies between $-14^{\circ} \mathrm{C}$ and $+37^{\circ} \mathrm{C}$. Any ecological condition that could affect vine growing negatively had not been occurred in the experimental period as seen in Table 2.1.

Soil analyses were done in Eğirdir Fruit Research Station Soil analyses laboratories. Soil analyses report is in Table 2.2.

\subsection{Plant material}

11 grapevine cultivars (Burdur Dimriti, Siyah Gemre, Ak Gemre, Antep Büzgülü, Şam Büzgülü, Kuş Yüreği, Ak Dimrit, Senirkent Dimriti, Devegözü, Tilki Kuyruğu and Marzımat (Figs. 2.1.1-2.1.6). Quality features (phenology stages, soluble solid (\%), titratable acidity $(\mathrm{g} / \mathrm{l})$, yield

Table 2.1. Some climatic data of the experiment area.

\begin{tabular}{|c|c|c|c|c|c|}
\hline Year & $\begin{array}{c}\text { Avg. } \\
\text { Temperature } \\
\left({ }^{\circ} \mathbf{C}\right)\end{array}$ & $\begin{array}{c}\text { Min. } \\
\text { Temperature } \\
\left({ }^{\circ} \mathbf{C}\right)\end{array}$ & $\begin{array}{c}\text { Max. } \\
\text { Temperature } \\
\left({ }^{\circ} \mathbf{C}\right)\end{array}$ & $\begin{array}{c}\text { Avg. } \\
\text { Precipitation } \\
(\mathbf{m m})\end{array}$ & $\begin{array}{c}\text { Avg. } \\
\text { Humidity }\end{array}$ \\
\hline Average & 12.2 & -14.9 & 38.6 & 764 & 66.5 \\
\hline 2011 & 11.9 & -8.2 & 37.4 & 669 & 66.3 \\
\hline 2012 & 12.9 & -12.7 & 38.6 & 622 & 68.9 \\
\hline 2013 & 13.1 & -7.9 & 34.1 & 654 & 63.7 \\
\hline 2014 & 13.4 & -6.3 & 36.1 & 669 & 70.9 \\
\hline
\end{tabular}

Table 2.2. Soil analyses of the experiment area.

\begin{tabular}{|c|c|c|}
\hline \multicolumn{3}{|l|}{ Physical analyses } \\
\hline & Texture & Loam \\
\hline & $\mathrm{pH}$ & 7.78 \\
\hline & Lime (\%) & 9.8 \\
\hline & Saturation (\%) & 49 \\
\hline \multicolumn{3}{|l|}{ Chemical analyses } \\
\hline & Organic matter $(\%)$ & 2.8 \\
\hline & $\mathrm{N}(\mathrm{ppm})$ & 1617 \\
\hline & $\mathrm{P}(\mathrm{ppm})$ & 4.52 \\
\hline & $\mathrm{K}(\mathrm{ppm})$ & 221.1 \\
\hline & $\mathrm{Ca}(\mathrm{ppm})$ & 4679 \\
\hline & $\mathrm{Mg}(\mathrm{ppm})$ & 492 \\
\hline & $\mathrm{Na}(\mathrm{ppm})$ & 13.48 \\
\hline & $\mathrm{Fe}(\mathrm{ppm})$ & 13.64 \\
\hline & $\mathrm{Cu}(\mathrm{ppm})$ & 4.23 \\
\hline & Mn (ppm) & 6.25 \\
\hline & $\mathrm{Zn}(\mathrm{ppm})$ & 1.04 \\
\hline
\end{tabular}

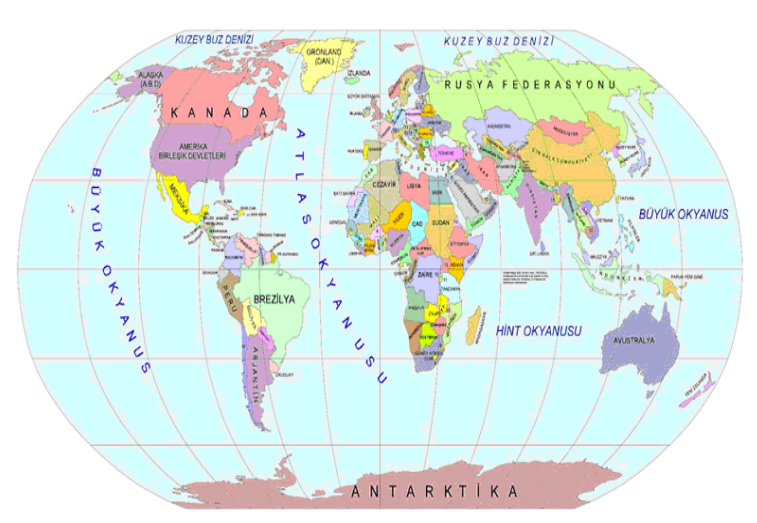

Figure 2.1. World Map.

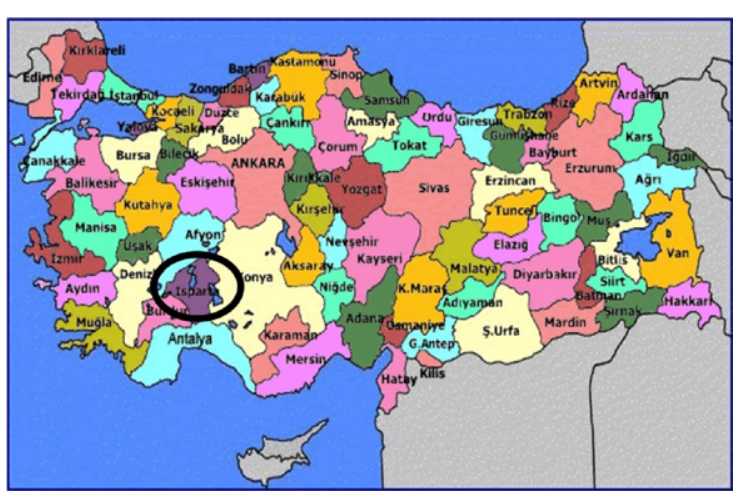

Figure 2.2. Turkey Map and Isparta City. 
(kg/vine), average bunch weight (g), must ratio (\%) data had been obtained in vineyard for two years. Samples of the grape cultivars used in this study were obtained from Eğirdir Fruit Station research vineyard in 2013, at the same developmental stage when harvested. The fully ripened and matured fruits were harvested in September and they were stored for about 7 months at $-80^{\circ} \mathrm{C}$.

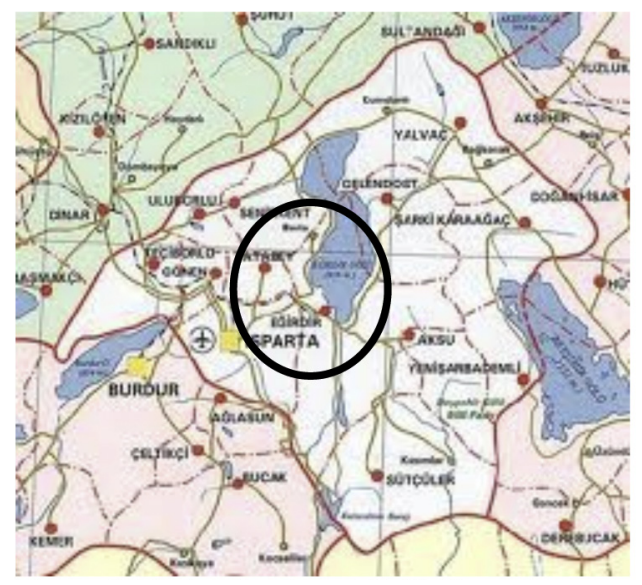

Figure 2.3. Lakes Region and Eğirdir town.

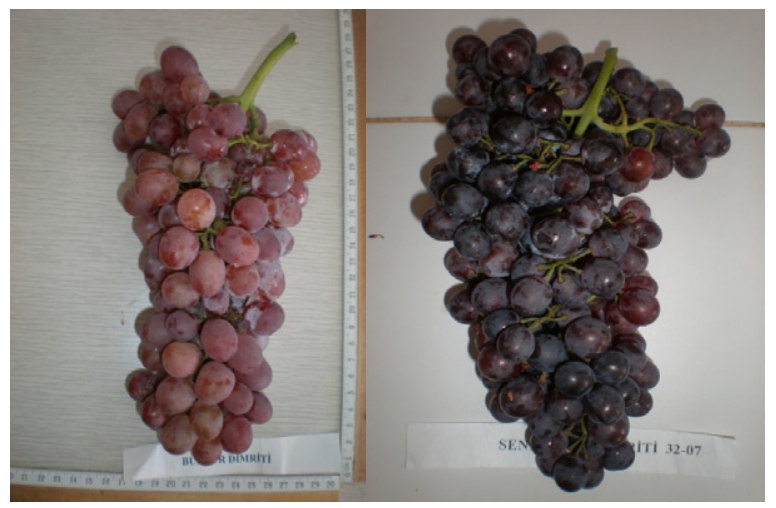

Figure 2.1.1. Burdur Dimriti and Senirkent Dimriti varieties.

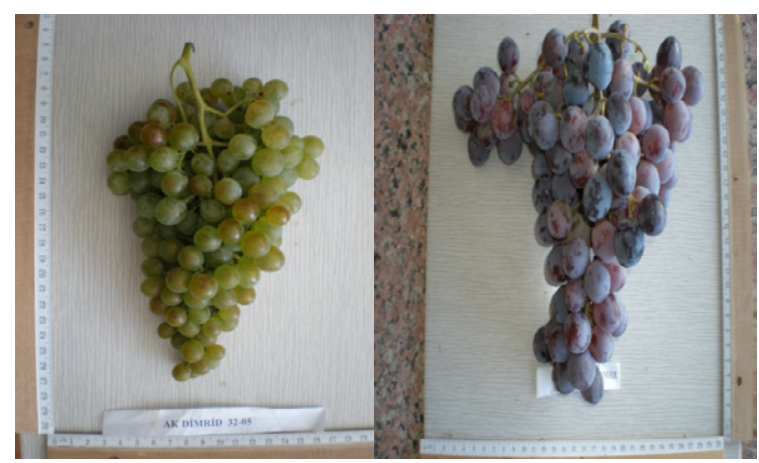

Figure 2.1.2. Ak Dimrit and Siyah Gemre varieties.

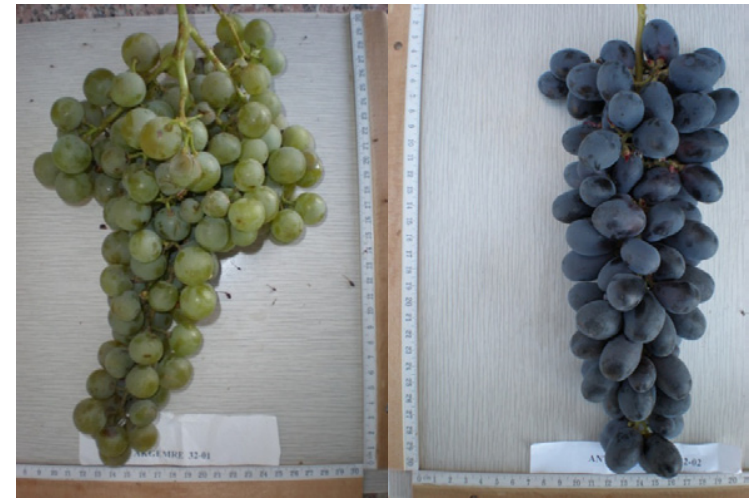

Figure 2.1.3. Ak Gemre and Antep Büzgülü varieties.

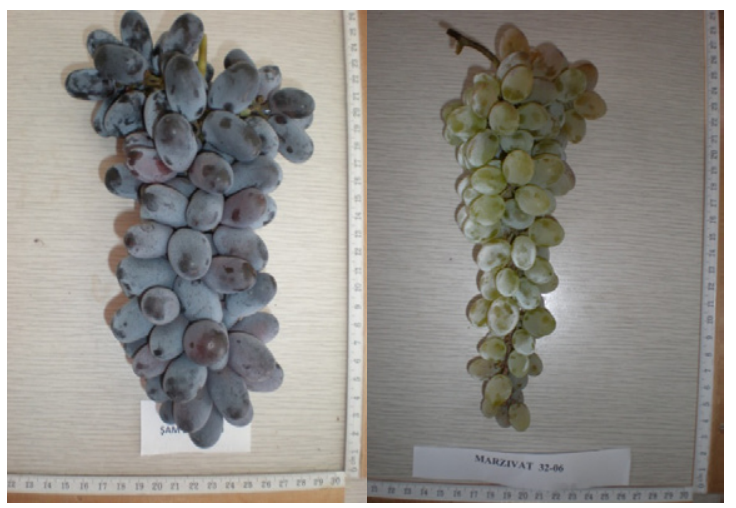

Figure 2.1.4. Şam Büzgülü and Marzımat varieties.

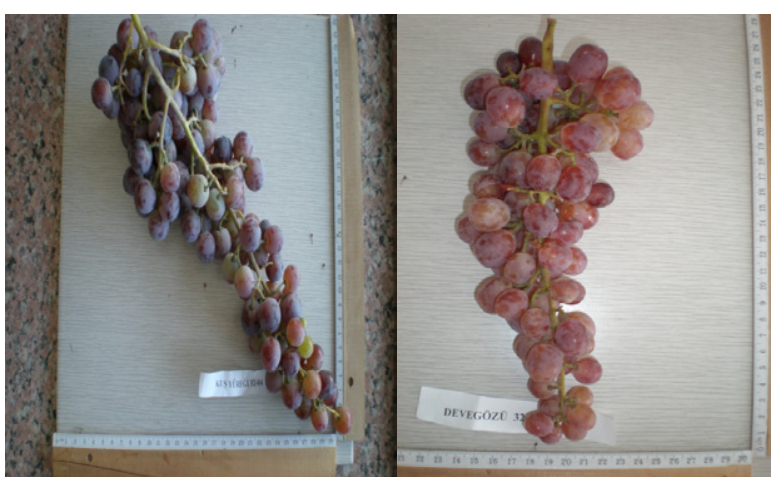

Figure 2.1.5. Kuş Yüreği and Devegözü varieties.

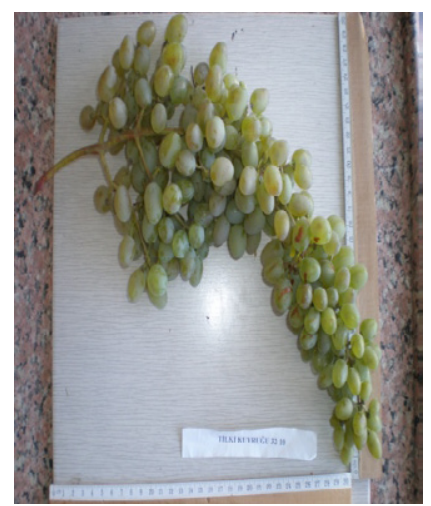

Figure. 2.1.6. Tilki Kuyruğu variety. 


\subsection{Experimental methods}

Quality analyses were done in the vegetation period. Phenology stages, yield and quality properties data had been obtained in vineyard. Soluble solid (\%), titratable acidity ( $\mathrm{g} / \mathrm{l})$, yield ( $\mathrm{kg} / \mathrm{vine})$ were determined. Also climate data for 2013 year in the study had been recorded. Phenolic compound analyses were done in Ege University Faculty of Pharmacy Pharmaceutical Sciences Research Centre laboratories in 2014 year by HPLC. Thermo Scientific brand Accela model liquid chromatography device was used in the analyses. Device has a pump, photo diod array detector (PDA), $25 \mu l$ loop volume automatic sampler, and integrated collar oven to the automatic sampler). Separation was done on a ACE type collar size $250 \mathrm{~mm} \times 4.6 \mathrm{~mm} \times 5$ microns. Solvent A was consisting of \% 0.25 trifloroacetic acid and water solvent $\mathrm{b}$ was consisting of acetonitril. Acetonitril in HPLC purity trifloroacetic acid in spectro purity, water in HPLC purity, methanol $(\mathrm{MeOH})$ in HPLC purity were used in analyses. All standards were weighed $1 \mathrm{mg}$ and they were solved in $1 \mathrm{~mL} \mathrm{MeOH}$ main reserves $(1000 \mu \mathrm{g}$ / $\mathrm{ml}$ ) were stored in $4^{\circ} \mathrm{C}$ for one month and dilutions were made with $\mathrm{MeOH}$ (Table 2.2.1).

\subsection{Polyphenols extraction}

Polyphenols extraction grapes, in three replicated each, was extracted by grinding the $2 \mathrm{~g}$ sample $1 \mathrm{~min}$ at $20,000 \mathrm{rpm}$ in a blender with $2 \mathrm{ml}$ of acidified methanol $(85: 15 \mathrm{v} / \mathrm{v}$, $\mathrm{MeOH}: \mathrm{HCl}$ ) and stored for one night. The homogenate was centrifuged at $4500 \mathrm{rpm}$ for $10 \mathrm{~min}$ at $25^{\circ} \mathrm{C}$. The extract was separated and the residual tissue was re-extracted until the extraction solvents became colorless. The filtrates were combined in a total extract and dried using for 2 days time in lyophilizator. This producer was also done with acetonitril (ACN) but good results were not obtained with (ACN), for that reason $\mathrm{MeOH}$ extraction systems which resulted better were used in the analyses.

\subsection{Separation of phenolic acids}

Samples were filtered from $45 \mu \mathrm{m}$ and a sample with $10 \mu \mathrm{L}$ injection volume was injected into HPLC and

Table 2.2.1. Standards used in the analyses.

\begin{tabular}{|l|l|}
\hline Standart Number & Standart Name \\
\hline STD1 & Gallic Acid \\
\hline STD2 & Epigallokatechin \\
\hline STD3 & Katechin \\
\hline STD4 & Epigallokatechin-3-gallat \\
\hline STD5 & Ferulic Acid \\
\hline STD6 & Myricetin \\
\hline STD7 & Resveratrol \\
\hline STD8 & Quercetin \\
\hline STD9 & Kamferol \\
\hline STD10 & Kafeic Acid \\
\hline STD11 & p-Kumaric Acid \\
\hline STD12 & Rutinhidrate \\
\hline
\end{tabular}

mobile phase gradient was used with flow $1000 \mu \mathrm{L} / \mathrm{min}$. (Tables 2.4.1, 2.4.2). Separation was done on an ACE type collar size $250 \mathrm{~mm} \times 4.6 \mathrm{~mm} \times 5$ microns, a wavelength of between 254 and $280 \mathrm{~nm}$. For each injection and 10,000 $\mu \mathrm{L}$ extract was used at a working temperature of $25^{\circ} \mathrm{C}$. 2 different method were used in the determination seen on (Tables 2.4.1, 2.4.2).

\section{Results and discussion}

\subsection{Yield and quality characteristic of varieties}

Grape varieties which were evaluated in the study, all phonological stages were determined. Buds burst time, Full blossom time, verasion period and harvest time were given in Table (3.1). All varieties buds burst time was mid of the April. The earliest varieties were Senirkent Dimridi, Ak Dimrit and Burdur Dimriti were determined. Full blossom time in all varieties were second and third week of the June in observation. Harvest time of varieties were late August and September and beginning of the October. Generally all varieties are harvested in September.

Among the 11 variety yield and quality properties data had been obtained in vineyard. Yield (kg/vine), soluble solid $(\%)$, total titratable acidity $(\mathrm{g} / \mathrm{l})$, Average bunch weight $(\mathrm{g})$, must ratio $(\%)$ was determined. Data were given in Table 3.1.2. Highest total yield were evaluated from Burdur Dimridi, Şam Büzgülü and Antep Büzgülü varieties. Highest bunch weight was evaluated from Ak Gemre and Tilki Kuyruğu varieties. All varieties were good in must ratio. Generally must ratios were at $\% 60$ or more. Ak Gemre, Marzımat, Kuş Yüreği and Dimrit varieties (Burdur Dimriti, Senirkent Dimriti, Ak Dimrit) have high must ratios. Titratable acidity had varied 4.6-6.9 g/l. Some varieties were evaluated for good for must ratios and yield characterics.

Table 2.4.1. Gradient solvents program used for HPLC separation (first method).

\begin{tabular}{|l|c|c|c|c|c|}
\hline $\begin{array}{l}\text { Solvent } \\
\text { type }\end{array}$ & $\begin{array}{c}\text { Time } \\
(\mathbf{m i n})\end{array}$ & $\begin{array}{c}\text { Time } \\
(\mathbf{m i n})\end{array}$ & $\begin{array}{c}\text { Time } \\
(\mathbf{m i n})\end{array}$ & $\begin{array}{c}\text { Time } \\
(\mathbf{m i n})\end{array}$ & Flow rate \\
\hline $\begin{array}{l}\text { Solvent } \\
\text { A\% }\end{array}$ & 10 & 20 & 70 & 10 & 1000 \\
\hline $\begin{array}{l}\text { Solvent } \\
\text { B\% }\end{array}$ & 90 & 80 & 30 & 90 & 1000 \\
\hline
\end{tabular}

Solvent A: acetonitril ACN with \% 0.25 trifloroacetic acid TFA.

Solvent B: water with $\% 0.25$ with trifloroacetic acid TFA.

Table 2.4.2. Gradient solvents program used for HPLC separation (second method).

\begin{tabular}{|c|c|c|c|c|c|}
\hline $\begin{array}{c}\text { Solvent } \\
\text { type }\end{array}$ & $\begin{array}{l}\text { Time } \\
(\mathbf{m i n})\end{array}$ & $\begin{array}{l}\text { Time } \\
\text { (min) }\end{array}$ & $\begin{array}{l}\text { Time } \\
(\mathbf{m i n})\end{array}$ & $\begin{array}{l}\text { Time } \\
\text { (min) }\end{array}$ & $\begin{array}{l}\text { Flow } \\
\text { rate }\end{array}$ \\
\hline & 0 & 4.00 & 13.00 & 15.00 & $\mu \mathrm{L} / \mathrm{min}$ \\
\hline $\begin{array}{l}\text { Solvent } \\
\text { A\% }\end{array}$ & 10 & 20 & 70 & 10 & 1000 \\
\hline $\begin{array}{l}\text { Solvent } \\
\text { B\% }\end{array}$ & 90 & 80 & 30 & 90 & 1000 \\
\hline
\end{tabular}

Solvent A: acetonitril ACN with $\% 0.25$ trifloroacetic acid TFA Solvent B: water with $\% 0.25$ with trifloroacetic acid TFA. 
Table 3.1. Phenologic stages of varieties.

\begin{tabular}{|l|c|c|c|c|}
\hline Variety & $\begin{array}{c}\text { Bud } \\
\text { burst } \\
\text { time }\end{array}$ & $\begin{array}{c}\text { Flower } \\
\text { time }\end{array}$ & $\begin{array}{c}\text { Berry } \\
\text { colour set } \\
\text { time }\end{array}$ & $\begin{array}{c}\text { Harvest } \\
\text { time }\end{array}$ \\
\hline Ak Gemre & 18.04 & 15.06 & 26.08 & 30.09 \\
\hline $\begin{array}{l}\text { Antep } \\
\text { Büzgülü }\end{array}$ & 22.04 & 14.06 & 06.08 & 26.09 \\
\hline $\begin{array}{l}\text { Şam } \\
\text { Büzgülü }\end{array}$ & 22.04 & 16.06 & 02.08 & 17.09 \\
\hline Kuş Böreği & 21.04 & 16.06 & 25.08 & 30.09 \\
\hline $\begin{array}{l}\text { Ak Dimrit } \\
17.04\end{array}$ & 13.06 & 26.08 & 12.09 \\
\hline $\begin{array}{l}\text { Senirkent } \\
\text { Dimridi }\end{array}$ & 18.04 & 12.06 & 30.07 & 25.08 \\
\hline Devegözü & 24.04 & 15.06 & 19.08 & 02.10 \\
\hline $\begin{array}{l}\text { Tilki } \\
\text { Kuyruğu }\end{array}$ & 20.04 & 15.06 & 25.08 & 03.10 \\
\hline $\begin{array}{l}\text { Burdur } \\
\text { Dimriti }\end{array}$ & 20.04 & 12.06 & 29.07 & 27.08 \\
\hline $\begin{array}{l}\text { Siyah } \\
\text { Gemre }\end{array}$ & 19.04 & 10.06 & 10.08 & 25.09 \\
\hline Marzimat & 21.04 & 14.06 & 20.08 & 26.09 \\
\hline
\end{tabular}

Table 3.1.2. Quality features of varieties.

\begin{tabular}{|l|c|c|c|c|c|}
\hline Variety & $\begin{array}{c}\text { Yield } \\
\text { kg/vine }\end{array}$ & $\begin{array}{c}\text { Avg. } \\
\text { bunch } \\
\text { weight } \\
(\mathbf{g})\end{array}$ & $\begin{array}{c}\text { Total } \\
\text { solub } \\
(\%)\end{array}$ & $\begin{array}{c}\text { Acidity } \\
(\mathbf{g} / \mathbf{l})\end{array}$ & $\begin{array}{c}\text { Must } \\
\text { ratio } \\
(\%)\end{array}$ \\
\hline $\begin{array}{l}\text { Ak } \\
\text { Gemre }\end{array}$ & 12.25 & 640 & 15.0 & 5.6 & 58 \\
\hline $\begin{array}{l}\text { Antep } \\
\text { Büzgülü }\end{array}$ & 18.66 & 533 & 17.6 & 6.7 & 56 \\
\hline $\begin{array}{l}\text { Şam } \\
\text { Büzgülü }\end{array}$ & 21.78 & 622 & 17.8 & 5.3 & 57 \\
\hline $\begin{array}{l}\text { Kuş } \\
\text { Böreği }\end{array}$ & 16.10 & 600 & 16.4 & 4.8 & 61 \\
\hline $\begin{array}{l}\text { Ak } \\
\text { Dimrit }\end{array}$ & 11.30 & 230 & 15.5 & 6.5 & 59 \\
\hline $\begin{array}{l}\text { Senirkent } \\
\text { Dimriti }\end{array}$ & 12.40 & 322 & 15.9 & 6.6 & 63 \\
\hline Devegözü & 7.50 & 368 & 15.4 & 6.1 & 58 \\
\hline $\begin{array}{l}\text { Tilki } \\
\text { Kuyruğu }\end{array}$ & 10.66 & 740 & 17.0 & 4.6 & 60 \\
\hline $\begin{array}{l}\text { Burdur } \\
\text { Dimriti }\end{array}$ & 21.60 & 375 & 18.5 & 6.9 & 62 \\
\hline $\begin{array}{l}\text { Siyah } \\
\text { Gemre }\end{array}$ & 16.45 & 383 & 17.2 & 6.3 & 54 \\
\hline Marzımat & 12.35 & 365 & 16.8 & 5.8 & 59 \\
\hline
\end{tabular}

\subsection{Phenolic composition results}

Phenolic compositions were determined by two different methods and extraction was done by $\mathrm{MeOH}$ and $\mathrm{HCl}$. $20 \mathrm{~min}$ method gave better results than $15 \mathrm{~min}$ results. 20 min HPLC and meoh and $\mathrm{HCl}$ extraction methods were given on tables below.

\subsubsection{Gallic acid}

Gallic acid values and reaction equation results are given in Table 3.2.1 and Fig. 3.2.1. Highest gallic acid values were evaluated from Siyah Gemre, Antep Büzgülü, Şam Büzgülü and Ak Dimrit varieties and the other varieties gallic acid values were determined less or not found (NF).

Table 3.2.1. Average gallic acid results according to variety.

\begin{tabular}{|l|c|c|}
\hline Ak Gemre $\mathrm{HCl}$ & $\mathrm{NF}$ & $\mu \mathrm{g} / \mathrm{mL}$ \\
\hline Ak Gemre MeOH & $\mathrm{NF}$ & $\mu \mathrm{g} / \mathrm{mL}$ \\
\hline Ak Dimrit $\mathrm{HCl}$ & $\mathrm{NF}$ & $\mu \mathrm{g} / \mathrm{mL}$ \\
\hline Ak Dimrit MeOH & 0,425 & $\mu \mathrm{g} / \mathrm{mL}$ \\
\hline Antep Büzgülü $\mathrm{HCl}$ & 0,076 & $\mu \mathrm{g} / \mathrm{mL}$ \\
\hline Antep Büzgülü MeOH & $\mathrm{NF}$ & $\mu \mathrm{g} / \mathrm{mL}$ \\
\hline Burdur Dimriti $\mathrm{HCl}$ & $\mathrm{NF}$ & $\mu \mathrm{g} / \mathrm{mL}$ \\
\hline Burdur Dimriti MeOH & $\mathrm{NF}$ & $\mu \mathrm{g} / \mathrm{mL}$ \\
\hline Devegözü $\mathrm{HCl}$ & $\mathrm{NF}$ & $\mu \mathrm{g} / \mathrm{mL}$ \\
\hline Devegözü MeOH & $\mathrm{NF}$ & $\mu \mathrm{g} / \mathrm{mL}$ \\
\hline Kuş Yüreği $\mathrm{HCl}$ & $\mathrm{NF}$ & $\mu \mathrm{g} / \mathrm{mL}$ \\
\hline Kuş Yüreği MeOH & 0,220 & $\mu \mathrm{g} / \mathrm{mL}$ \\
\hline Marzımat $\mathrm{HCl}$ & $\mathrm{NF}$ & $\mu \mathrm{g} / \mathrm{mL}$ \\
\hline Marzımat MeOH & $\mathrm{NF}$ & $\mu \mathrm{g} / \mathrm{mL}$ \\
\hline Ak Gemre $20 \mu \mathrm{g} / \mathrm{mL} \mathrm{HCl}$ & 1,586 & $\mu \mathrm{g} / \mathrm{mL}$ \\
\hline Ak Gemre $20 \mu \mathrm{g} / \mathrm{mL} \mathrm{MeOH}$ & 28,566 & $\mu \mathrm{g} / \mathrm{mL}$ \\
\hline Ak Dimrit $20 \mu \mathrm{g} / \mathrm{mL} \mathrm{HCl}$ & 3,269 & $\mu \mathrm{g} / \mathrm{mL}$ \\
\hline Ak Dimrit $20 \mu \mathrm{g} / \mathrm{mL} \mathrm{MeOH}$ & 30,221 & $\mu \mathrm{g} / \mathrm{mL}$ \\
\hline Antep Büzgülü $20 \mu \mathrm{g} / \mathrm{mL} \mathrm{HCl}$ & 2,505 & $\mu \mathrm{g} / \mathrm{mL}$ \\
\hline Antep Büzgülü $20 \mu \mathrm{g} / \mathrm{mL} \mathrm{MeOH}$ & 28,902 & $\mu \mathrm{g} / \mathrm{mL}$ \\
\hline Burdur Dimriti $20 \mu \mathrm{g} / \mathrm{mL} \mathrm{HCl}$ & 2,489 & $\mu \mathrm{g} / \mathrm{mL}$ \\
\hline Burdur Dimriti $20 \mu \mathrm{g} / \mathrm{mL} \mathrm{MeOH}$ & 17,282 & $\mu \mathrm{g} / \mathrm{mL}$ \\
\hline Devegözü $20 \mu \mathrm{g} / \mathrm{mL} \mathrm{HCl}$ & 2,343 & $\mu \mathrm{g} / \mathrm{mL}$ \\
\hline Devegözü $20 \mu \mathrm{g} / \mathrm{mL} \mathrm{MeOH}$ & 28,022 & $\mu \mathrm{g} / \mathrm{mL}$ \\
\hline Kuş Yureği $20 \mu \mathrm{g} / \mathrm{mL} \mathrm{HCl}$ & 2,396 & $\mu \mathrm{g} / \mathrm{mL}$ \\
\hline Kuş Yüreği $20 \mu \mathrm{g} / \mathrm{mL} \mathrm{MeOH}$ & 17,795 & $\mu \mathrm{g} / \mathrm{mL}$ \\
\hline Marzımat $20 \mu \mathrm{g} / \mathrm{mL} \mathrm{HCl}$ & 4,932 & $\mu \mathrm{g} / \mathrm{mL}$ \\
\hline Marzımat $20 \mu \mathrm{g} / \mathrm{mL} \mathrm{MeOH}$ & 16,441 & $\mu \mathrm{g} / \mathrm{mL}$ \\
\hline Şam Büzgülü $20 \mu \mathrm{g} / \mathrm{mL} \mathrm{HCl}$ & 0,168 & $\mu \mathrm{g} / \mathrm{mL}$ \\
\hline Şam Büzgülü $20 \mu \mathrm{g} / \mathrm{mL} \mathrm{MeOH}$ & 28,469 & $\mu \mathrm{g} / \mathrm{mL}$ \\
\hline Senirkent Dimriti $20 \mu \mathrm{g} / \mathrm{mL} \mathrm{HCl}$ & 2,317 & $\mu \mathrm{g} / \mathrm{mL}$ \\
\hline Senirkent Dimriti $20 \mu \mathrm{g} / \mathrm{mL} \mathrm{MeOH}$ & 28,235 & $\mu \mathrm{g} / \mathrm{mL}$ \\
\hline Siyah Gemre $20 \mu \mathrm{g} / \mathrm{mL} \mathrm{HCl}$ & 2,42 & $\mu \mathrm{g} / \mathrm{mL}$ \\
\hline
\end{tabular}


Table 3.2.1. Continued.

\begin{tabular}{|l|c|c|}
\hline Siyah Gemre $20 \mu \mathrm{g} / \mathrm{mL} \mathrm{MeOH}$ & 28,202 & $\mu \mathrm{g} / \mathrm{mL}$ \\
\hline Tilki Kuyruğu $20 \mu \mathrm{g} / \mathrm{mL} \mathrm{HCl}$ & 2,317 & $\mu \mathrm{g} / \mathrm{mL}$ \\
\hline Tilki Kuyruğu $20 \mu \mathrm{g} / \mathrm{mL} \mathrm{MeOH}$ & 28,356 & $\mu \mathrm{g} / \mathrm{mL}$ \\
\hline Şam Büzgülü HCl & 2,706 & $\mu \mathrm{g} / \mathrm{mL}$ \\
\hline Şam Büzgülü MeOH & $\mathrm{NF}$ & $\mu \mathrm{g} / \mathrm{mL}$ \\
\hline Senirkent DimritiHCl & 5,282 & $\mu \mathrm{g} / \mathrm{mL}$ \\
\hline Senirkent Dimriti MeOH & $\mathrm{NF}$ & $\mu \mathrm{g} / \mathrm{mL}$ \\
\hline Siyah Gemre $\mathrm{HCl}$ & $\mathrm{NF}$ & $\mu \mathrm{g} / \mathrm{mL}$ \\
\hline Siyah Gemre MeOH & 0,228 & $\mu \mathrm{g} / \mathrm{mL}$ \\
\hline STD1:20ppm_ & 21,876 & $\mu \mathrm{g} / \mathrm{mL}$ \\
\hline Tilki Kuyruğu HCl & $\mathrm{NF}$ & $\mu \mathrm{g} / \mathrm{mL}$ \\
\hline Tilki Kuyruğu MeOH & $\mathrm{NF}$ & $\mu \mathrm{g} / \mathrm{mL}$ \\
\hline
\end{tabular}

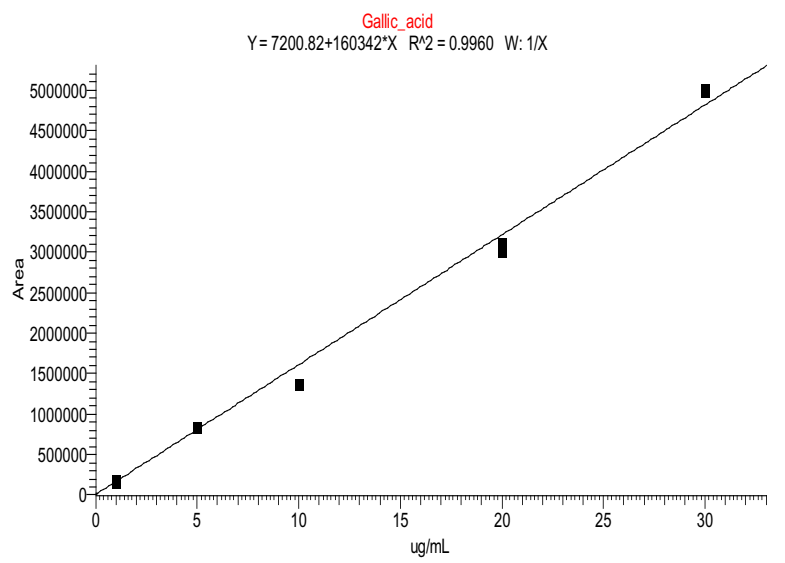

Figure 3.2.1. Gallic acid reaction equation.

\subsubsection{Epigallocatechin}

Epigallokatechin values and reaction equation results are given in Table 3.2.2 and Fig. 3.2.2. Highest Epigallocatechin values were evaluated from Siyah Gemre, Senirkent Dimriti, Ak Gemre, Şam Büzgülü and Ak Dimrit for other varieties epigallocatechin values were determined less or not found (NF).

Table 3.2.2. Epigallocatechin results according to variety.

\begin{tabular}{|l|c|c|}
\hline Ak Gemre $\mathrm{HCl}$ & $\mathrm{NF}$ & $\mu \mathrm{g} / \mathrm{mL}$ \\
\hline Ak Gemre $\mathrm{MeOH}$ & $\mathrm{NF}$ & $\mu \mathrm{g} / \mathrm{mL}$ \\
\hline Ak Dimrit $\mathrm{HCl}$ & $\mathrm{NF}$ & $\mu \mathrm{g} / \mathrm{mL}$ \\
\hline Ak Dimrit $\mathrm{MeOH}$ & $\mathrm{NF}$ & $\mu \mathrm{g} / \mathrm{mL}$ \\
\hline Antep Büzgülü $\mathrm{HCl}$ & $\mathrm{NF}$ & $\mu \mathrm{g} / \mathrm{mL}$ \\
\hline Antep Büzgülü $\mathrm{MeOH}$ & $\mathrm{NF}$ & $\mu \mathrm{g} / \mathrm{mL}$ \\
\hline Burdur Dimriti $\mathrm{HCl}$ & $\mathrm{NF}$ & $\mu \mathrm{g} / \mathrm{mL}$ \\
\hline Burdur Dimriti $\mathrm{MeOH}$ & $\mathrm{NF}$ & $\mu \mathrm{g} / \mathrm{mL}$ \\
\hline Devegözü $\mathrm{HCl}$ & $\mathrm{NF}$ & $\mu \mathrm{g} / \mathrm{mL}$ \\
\hline Devegözü $\mathrm{MeOH}$ & $\mathrm{NF}$ & $\mu \mathrm{g} / \mathrm{mL}$ \\
\hline Kuş Yüreği $\mathrm{HCl}$ & $\mathrm{NF}$ & $\mu \mathrm{g} / \mathrm{mL}$ \\
\hline
\end{tabular}

\begin{tabular}{|c|c|c|}
\hline Kis Yüreği $\mathrm{MeOH}$ & NF & $\mu \mathrm{g} / \mathrm{mL}$ \\
\hline Marzımat $\mathrm{HCl}$ & NF & $\mu \mathrm{g} / \mathrm{mL}$ \\
\hline Marzımat $\mathrm{MeOH}$ & NF & $\mu \mathrm{g} / \mathrm{mL}$ \\
\hline Ak Gemre $20 \mu \mathrm{g} / \mathrm{mL} \mathrm{HCl}$ & NF & $\mu \mathrm{g} / \mathrm{mL}$ \\
\hline Ak Gemre $20 \mu \mathrm{g} / \mathrm{mL} \mathrm{MeOH}$ & 30,530 & $\mu \mathrm{g} / \mathrm{mL}$ \\
\hline Ak Dimrit $20 \mu \mathrm{g} / \mathrm{mL} \mathrm{HCl}$ & NF & $\mu \mathrm{g} / \mathrm{mL}$ \\
\hline Ak Dimrit $20 \mu \mathrm{g} / \mathrm{mL} \mathrm{MeOH}$ & 29,661 & $\mu \mathrm{g} / \mathrm{mL}$ \\
\hline Antep Büzgülü $20 \mu \mathrm{g} / \mathrm{mL} \mathrm{HCl}$ & 4,453 & $\mu \mathrm{g} / \mathrm{mL}$ \\
\hline Antep Büzgülü $20 \mu \mathrm{g} / \mathrm{mL} \mathrm{MeOH}$ & 28,741 & $\mu \mathrm{g} / \mathrm{mL}$ \\
\hline Burdur Dimriti $20 \mu \mathrm{g} / \mathrm{mL} \mathrm{HCl}$ & 1,642 & $\mu \mathrm{g} / \mathrm{mL}$ \\
\hline Burdur Dimriti $20 \mu \mathrm{g} / \mathrm{mL} \mathrm{MeOH}$ & 18,983 & $\mu \mathrm{g} / \mathrm{mL}$ \\
\hline Devegözü $20 \mu \mathrm{g} / \mathrm{mL} \mathrm{HCl}$ & NF & $\mu \mathrm{g} / \mathrm{mL}$ \\
\hline Devegözü $20 \mu \mathrm{g} / \mathrm{mL} \mathrm{MeOH}$ & 28,281 & $\mu \mathrm{g} / \mathrm{mL}$ \\
\hline Kuş Yureği $20 \mu \mathrm{g} / \mathrm{mL} \mathrm{HCl}$ & NF & $\mu \mathrm{g} / \mathrm{mL}$ \\
\hline Kuş Yüreği $20 \mu \mathrm{g} / \mathrm{mL} \mathrm{MeOH}$ & 18,391 & $\mu \mathrm{g} / \mathrm{mL}$ \\
\hline Marzımat $20 \mu \mathrm{g} / \mathrm{mL} \mathrm{HCl}$ & 4,655 & $\mu \mathrm{g} / \mathrm{mL}$ \\
\hline Marzımat $20 \mu \mathrm{g} / \mathrm{mL} \mathrm{MeOH}$ & 17,219 & $\mu \mathrm{g} / \mathrm{mL}$ \\
\hline Şam Büzgülü $20 \mu \mathrm{g} / \mathrm{mL} \mathrm{HCl}$ & NF & $\mu \mathrm{g} / \mathrm{mL}$ \\
\hline Şam Büzgülü $20 \mu \mathrm{g} / \mathrm{mL} \mathrm{MeOH}$ & 29,069 & $\mu \mathrm{g} / \mathrm{mL}$ \\
\hline Senirkent Dimriti $20 \mu \mathrm{g} / \mathrm{mL} \mathrm{HCl}$ & NF & $\mu \mathrm{g} / \mathrm{mL}$ \\
\hline Senirkent Dimriti $20 \mu \mathrm{g} / \mathrm{mL} \mathrm{MeOH}$ & 33,279 & $\mu \mathrm{g} / \mathrm{mL}$ \\
\hline Siyah Gemre $20 \mu \mathrm{g} / \mathrm{mL} \mathrm{HCl}$ & 2,473 & $\mu \mathrm{g} / \mathrm{mL}$ \\
\hline Siyah Gemre $20 \mu \mathrm{g} / \mathrm{mL} \mathrm{MeOH}$ & 55,896 & $\mu \mathrm{g} / \mathrm{mL}$ \\
\hline 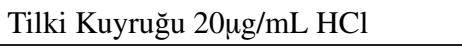 & NF & $\mu \mathrm{g} / \mathrm{mL}$ \\
\hline Tilki Kuyruğu $20 \mu \mathrm{g} / \mathrm{mL} \mathrm{MeOH}$ & 27,850 & $\mu \mathrm{g} / \mathrm{mL}$ \\
\hline Şam Büzgülü HCl & NF & $\mu \mathrm{g} / \mathrm{mL}$ \\
\hline Şam Büzgülü MeOH & NF & $\mu \mathrm{g} / \mathrm{mL}$ \\
\hline Senirkent DimritiHCl & NF & $\mu \mathrm{g} / \mathrm{mL}$ \\
\hline Senirkent Dimriti MeOH & NF & $\mu \mathrm{g} / \mathrm{mL}$ \\
\hline Siyah Gemre $\mathrm{HCl}$ & NF & $\mu \mathrm{g} / \mathrm{mL}$ \\
\hline Siyah Gemre $\mathrm{MeOH}$ & NF & $\mu \mathrm{g} / \mathrm{mL}$ \\
\hline STD1:20ppm_ & 20,967 & $\mu \mathrm{g} / \mathrm{mL}$ \\
\hline Tilki Kuyruğu HCl & NF & $\mu \mathrm{g} / \mathrm{mL}$ \\
\hline Tilki Kuyruğu MeOH & NF & $\mu \mathrm{g} / \mathrm{mL}$ \\
\hline
\end{tabular}

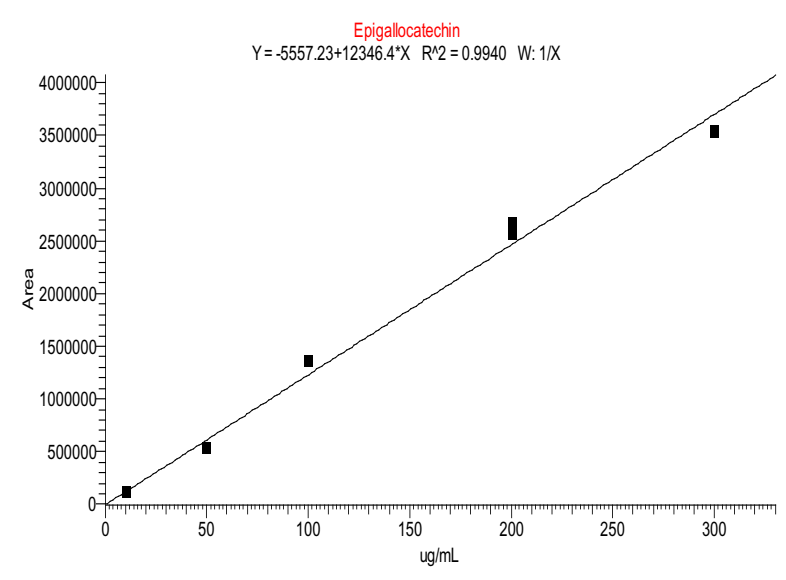

Figure 3.2.2. Epigallocatechin reaction equation. 


\subsubsection{Catechin}

Katechin values and reaction equation results are given in Table 3.2.3 and Fig. 3.2.3. Highest catechin values were evaluated from Kuş Yüregi and Burdur Dimrit varieties other varieties values were determined less or not found (NF).

Table 3.2.3. Katechin results according to variety.

\begin{tabular}{|c|c|c|}
\hline Ak Gemre $\mathrm{HCl}$ & 0,186 & $\mu \mathrm{g} / \mathrm{mL}$ \\
\hline Ak Gemre $\mathrm{MeOH}$ & 1,242 & $\mu \mathrm{g} / \mathrm{mL}$ \\
\hline Ak Dimrit $\mathrm{HCl}$ & NF & $\mu \mathrm{g} / \mathrm{mL}$ \\
\hline Ak Dimrit $\mathrm{MeOH}$ & 9,383 & $\mu \mathrm{g} / \mathrm{mL}$ \\
\hline Antep Büzgülü $\mathrm{HCl}$ & NF & $\mu \mathrm{g} / \mathrm{mL}$ \\
\hline Antep Büzgülü MeOH & NF & $\mu \mathrm{g} / \mathrm{mL}$ \\
\hline Burdur Dimriti $\mathrm{HCl}$ & NF & $\mu \mathrm{g} / \mathrm{mL}$ \\
\hline Burdur Dimriti MeOH & NF & $\mu \mathrm{g} / \mathrm{mL}$ \\
\hline Devegözü HCl & NF & $\mu \mathrm{g} / \mathrm{mL}$ \\
\hline Devegözü MeOH & NF & $\mu \mathrm{g} / \mathrm{mL}$ \\
\hline Kuş Yüreği HCl & NF & $\mu \mathrm{g} / \mathrm{mL}$ \\
\hline Kuş Yüreği MeOH & 11,650 & $\mu \mathrm{g} / \mathrm{mL}$ \\
\hline Marzımat $\mathrm{HCl}$ & NF & $\mu \mathrm{g} / \mathrm{mL}$ \\
\hline Marzımat $\mathrm{MeOH}$ & 1,783 & $\mu \mathrm{g} / \mathrm{mL}$ \\
\hline Ak Gemre $20 \mu \mathrm{g} / \mathrm{mL} \mathrm{HCl}$ & NF & $\mu \mathrm{g} / \mathrm{mL}$ \\
\hline Ak Gemre $20 \mu \mathrm{g} / \mathrm{mL} \mathrm{MeOH}$ & NF & $\mu \mathrm{g} / \mathrm{mL}$ \\
\hline Ak Dimrit $20 \mu \mathrm{g} / \mathrm{mL} \mathrm{HCl}$ & 0,414 & $\mu \mathrm{g} / \mathrm{mL}$ \\
\hline Ak Dimrit $20 \mu \mathrm{g} / \mathrm{mL} \mathrm{MeOH}$ & 19,953 & $\mu \mathrm{g} / \mathrm{mL}$ \\
\hline Antep Büzgülü $20 \mu \mathrm{g} / \mathrm{mL} \mathrm{HCl}$ & 1,007 & $\mu \mathrm{g} / \mathrm{mL}$ \\
\hline Antep Büzgülü $20 \mu \mathrm{g} / \mathrm{mL} \mathrm{MeOH}$ & 0,355 & $\mu \mathrm{g} / \mathrm{mL}$ \\
\hline Burdur Dimriti $20 \mu \mathrm{g} / \mathrm{mL} \mathrm{HCl}$ & NF & $\mu \mathrm{g} / \mathrm{mL}$ \\
\hline Burdur Dimriti $20 \mu \mathrm{g} / \mathrm{mL} \mathrm{MeOH}$ & 23,802 & $\mu \mathrm{g} / \mathrm{mL}$ \\
\hline Devegözü $20 \mu \mathrm{g} / \mathrm{mL} \mathrm{HCl}$ & 11,034 & $\mu \mathrm{g} / \mathrm{mL}$ \\
\hline Devegözü $20 \mu \mathrm{g} / \mathrm{mL} \mathrm{MeOH}$ & 16,501 & $\mu \mathrm{g} / \mathrm{mL}$ \\
\hline Kuş Yureği $20 \mu \mathrm{g} / \mathrm{mL} \mathrm{HCl}$ & NF & $\mu \mathrm{g} / \mathrm{mL}$ \\
\hline Kuş Yüreği $20 \mu \mathrm{g} / \mathrm{mL} \mathrm{MeOH}$ & 31,190 & $\mu \mathrm{g} / \mathrm{mL}$ \\
\hline Marzımat $20 \mu \mathrm{g} / \mathrm{mL} \mathrm{HCl}$ & 14,441 & $\mu \mathrm{g} / \mathrm{mL}$ \\
\hline Marzımat $20 \mu \mathrm{g} / \mathrm{mL} \mathrm{MeOH}$ & 22,361 & $\mu \mathrm{g} / \mathrm{mL}$ \\
\hline Şam Büzgülü $20 \mu \mathrm{g} / \mathrm{mL} \mathrm{HCl}$ & NF & $\mu \mathrm{g} / \mathrm{mL}$ \\
\hline Şam Büzgülü $20 \mu \mathrm{g} / \mathrm{mL} \mathrm{MeOH}$ & 15,917 & $\mu \mathrm{g} / \mathrm{mL}$ \\
\hline Senirkent Dimriti $20 \mu \mathrm{g} / \mathrm{mL} \mathrm{HCl}$ & NF & $\mu \mathrm{g} / \mathrm{mL}$ \\
\hline Senirkent Dimriti $20 \mu \mathrm{g} / \mathrm{mL} \mathrm{MeOH}$ & 16,439 & $\mu \mathrm{g} / \mathrm{mL}$ \\
\hline Siyah Gemre $20 \mu \mathrm{g} / \mathrm{mL} \mathrm{HCl}$ & 18,02 & $\mu \mathrm{g} / \mathrm{mL}$ \\
\hline Siyah Gemre $20 \mu \mathrm{g} / \mathrm{mL} \mathrm{MeOH}$ & 19,298 & $\mu \mathrm{g} / \mathrm{mL}$ \\
\hline Tilki Kuyruğu 20ug/mL HCl & NF & $\mu \mathrm{g} / \mathrm{mL}$ \\
\hline Tilki Kuyruğu $20 \mu \mathrm{g} / \mathrm{mL} \mathrm{MeOH}$ & 19,006 & $\mu \mathrm{g} / \mathrm{mL}$ \\
\hline Şam Büzgülü $\mathrm{HCl}$ & NF & $\mu \mathrm{g} / \mathrm{mL}$ \\
\hline Şam Büzgülü MeOH & NF & $\mu \mathrm{g} / \mathrm{mL}$ \\
\hline Senirkent DimritiHCl & NF & $\mu \mathrm{g} / \mathrm{mL}$ \\
\hline
\end{tabular}

\begin{tabular}{|l|c|c|}
\hline Senirkent Dimriti MeOH & 4,520 & $\mu \mathrm{g} / \mathrm{mL}$ \\
\hline Siyah Gemre HCl & NF & $\mu \mathrm{g} / \mathrm{mL}$ \\
\hline Siyah Gemre $\mathrm{MeOH}$ & 4,450 & $\mu \mathrm{g} / \mathrm{mL}$ \\
\hline STD1:20ppm_ & 20,376 & $\mu \mathrm{g} / \mathrm{mL}$ \\
\hline Tilki Kuyruğu HCl & 0,239 & $\mu \mathrm{g} / \mathrm{mL}$ \\
\hline Tilki Kuyruğu MeOH & NF & $\mu \mathrm{g} / \mathrm{mL}$ \\
\hline
\end{tabular}

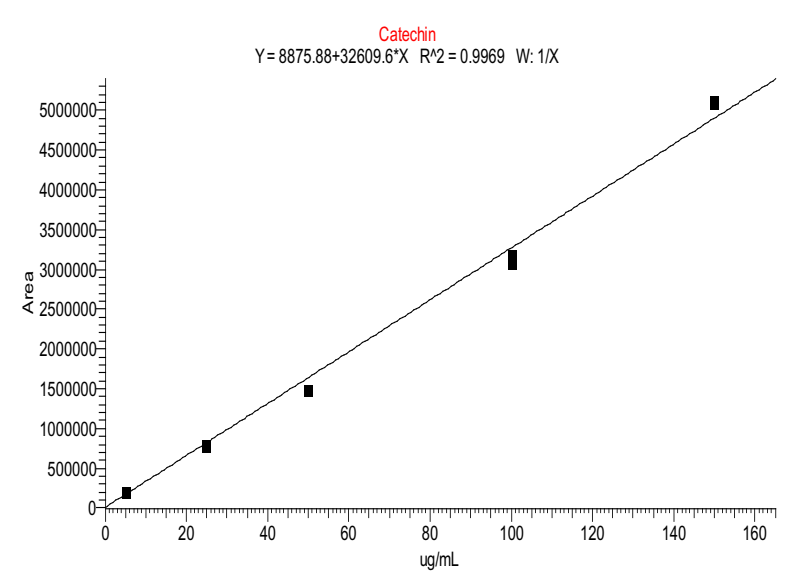

Figure 3.2.3. Catechin Reaction Equation.

\subsubsection{Epigallocatechin-3-Gallate}

Epigallocatechin-3-gallate values and reaction equation results are given in Table 3.2.4 and Fig. 3.2.4. Highest epigallocatechin-3-gallate values were evaluated from Senirkent Dimriti, Antep Büzgülü, Şam Büzgülü, Devegözü varieties. Senirkent Dimriti variety has a significant epigallocatechin-3-gallate content. The other varieties values were determined less or not found (NF).

Table 3.2.4. Epigallocatechin-3-gallate results according to variety

\begin{tabular}{|l|c|c|}
\hline Ak Gemre $\mathrm{HCl}$ & 1,532 & $\mu \mathrm{g} / \mathrm{mL}$ \\
\hline Ak Gemre $\mathrm{MeOH}$ & 0,925 & $\mu \mathrm{g} / \mathrm{mL}$ \\
\hline Ak Dimrit $\mathrm{HCl}$ & 3,289 & $\mu \mathrm{g} / \mathrm{mL}$ \\
\hline Ak Dimrit $\mathrm{MeOH}$ & 2,155 & $\mu \mathrm{g} / \mathrm{mL}$ \\
\hline Antep Büzgülü $\mathrm{HCl}$ & 0,693 & $\mu \mathrm{g} / \mathrm{mL}$ \\
\hline Antep Büzgülü $\mathrm{MeOH}$ & 3,055 & $\mu \mathrm{g} / \mathrm{mL}$ \\
\hline Burdur Dimriti $\mathrm{HCl}$ & 0,809 & $\mu \mathrm{g} / \mathrm{mL}$ \\
\hline Burdur Dimriti MeOH & $N F$ & $\mu \mathrm{g} / \mathrm{mL}$ \\
\hline Devegözü $\mathrm{HCl}$ & 0,675 & $\mu \mathrm{g} / \mathrm{mL}$ \\
\hline Devegözü $\mathrm{MeOH}$ & 1,162 & $\mu \mathrm{g} / \mathrm{mL}$ \\
\hline Kuş Yüreği $\mathrm{HCl}$ & 1,071 & $\mu \mathrm{g} / \mathrm{mL}$ \\
\hline Kuş Yüreği $\mathrm{MeOH}$ & 5,106 & $\mu \mathrm{g} / \mathrm{mL}$ \\
\hline Marzımat $\mathrm{HCl}$ & 0,902 & $\mu \mathrm{g} / \mathrm{mL}$ \\
\hline Marzımat $\mathrm{MeOH}$ & 0,756 & $\mu \mathrm{g} / \mathrm{mL}$ \\
\hline Ak Gemre $20 \mu \mathrm{g} / \mathrm{mL} \mathrm{HCl}$ & 66,447 & $\mu \mathrm{g} / \mathrm{mL}$ \\
\hline Ak Gemre $20 \mu \mathrm{g} / \mathrm{mL} \mathrm{MeOH}$ & 20,154 & $\mu \mathrm{g} / \mathrm{mL}$ \\
\hline
\end{tabular}


Table 3.2.4. Continued.

\begin{tabular}{|c|c|c|}
\hline Ak Dimrit $20 \mu \mathrm{g} / \mathrm{mL} \mathrm{HCl}$ & 56,150 & $\mu \mathrm{g} / \mathrm{mL}$ \\
\hline Ak Dimrit $20 \mu \mathrm{g} / \mathrm{mL} \mathrm{MeOH}$ & 19,653 & $\mu \mathrm{g} / \mathrm{mL}$ \\
\hline Antep Büzgülü $20 \mu \mathrm{g} / \mathrm{mL} \mathrm{HCl}$ & 91,059 & $\mu \mathrm{g} / \mathrm{mL}$ \\
\hline Antep Büzgülü $20 \mu \mathrm{g} / \mathrm{mL} \mathrm{MeOH}$ & 2,586 & $\mu \mathrm{g} / \mathrm{mL}$ \\
\hline Burdur Dimriti $20 \mu \mathrm{g} / \mathrm{mL} \mathrm{HCl}$ & 94,567 & $\mu \mathrm{g} / \mathrm{mL}$ \\
\hline Burdur Dimriti $20 \mu \mathrm{g} / \mathrm{mL} \mathrm{MeOH}$ & 30,472 & $\mu \mathrm{g} / \mathrm{mL}$ \\
\hline Devegözü $20 \mu \mathrm{g} / \mathrm{mL} \mathrm{HCl}$ & 89,054 & $\mu \mathrm{g} / \mathrm{mL}$ \\
\hline Devegözü $20 \mu \mathrm{g} / \mathrm{mL} \mathrm{MeOH}$ & 17,812 & $\mu \mathrm{g} / \mathrm{mL}$ \\
\hline Kuş Yureği $20 \mu \mathrm{g} / \mathrm{mL} \mathrm{HCl}$ & 96,227 & $\mu \mathrm{g} / \mathrm{mL}$ \\
\hline Kuş Yüreği $20 \mu \mathrm{g} / \mathrm{mL} \mathrm{MeOH}$ & 19,588 & $\mu \mathrm{g} / \mathrm{mL}$ \\
\hline Marzımat $20 \mu \mathrm{g} / \mathrm{mL} \mathrm{HCl}$ & 66,902 & $\mu \mathrm{g} / \mathrm{mL}$ \\
\hline Marzımat $20 \mu \mathrm{g} / \mathrm{mL} \mathrm{MeOH}$ & 19,589 & $\mu \mathrm{g} / \mathrm{mL}$ \\
\hline Şam Büzgülü $20 \mu \mathrm{g} / \mathrm{mL} \mathrm{HCl}$ & 97,014 & $\mu \mathrm{g} / \mathrm{mL}$ \\
\hline Şam Büzgülü $20 \mu \mathrm{g} / \mathrm{mL} \mathrm{MeOH}$ & 21,738 & $\mu \mathrm{g} / \mathrm{mL}$ \\
\hline Senirkent Dimriti $20 \mu \mathrm{g} / \mathrm{mL} \mathrm{HCl}$ & 101,466 & $\mu \mathrm{g} / \mathrm{mL}$ \\
\hline Senirkent Dimriti $20 \mu \mathrm{g} / \mathrm{mL} \mathrm{MeOH}$ & 18,185 & $\mu \mathrm{g} / \mathrm{mL}$ \\
\hline Siyah Gemre $20 \mu \mathrm{g} / \mathrm{mL} \mathrm{HCl}$ & 87,119 & $\mu \mathrm{g} / \mathrm{mL}$ \\
\hline Siyah Gemre $20 \mu \mathrm{g} / \mathrm{mL} \mathrm{MeOH}$ & 20,155 & $\mu \mathrm{g} / \mathrm{mL}$ \\
\hline 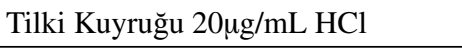 & 92,491 & $\mu \mathrm{g} / \mathrm{mL}$ \\
\hline Tilki Kuyruğu $20 \mu \mathrm{g} / \mathrm{mL} \mathrm{MeOH}$ & 20,071 & $\mu \mathrm{g} / \mathrm{mL}$ \\
\hline Şam Büzgülü $\mathrm{HCl}$ & 0,675 & $\mu \mathrm{g} / \mathrm{mL}$ \\
\hline Şam Büzgülü MeOH & 2,103 & $\mu \mathrm{g} / \mathrm{mL}$ \\
\hline Senirkent DimritiHCl & 2,030 & $\mu \mathrm{g} / \mathrm{mL}$ \\
\hline Senirkent Dimriti MeOH & 5,658 & $\mu \mathrm{g} / \mathrm{mL}$ \\
\hline Siyah Gemre $\mathrm{HCl}$ & NF & $\mu \mathrm{g} / \mathrm{mL}$ \\
\hline Siyah Gemre MeOH & $\mathrm{NF}$ & $\mu \mathrm{g} / \mathrm{mL}$ \\
\hline STD1:20ppm_ & 21,938 & $\mu \mathrm{g} / \mathrm{mL}$ \\
\hline Tilki Kuyruğu HCl & 0,812 & $\mu \mathrm{g} / \mathrm{mL}$ \\
\hline Tilki Kuyruğu MeOH & 0,746 & $\mu \mathrm{g} / \mathrm{mL}$ \\
\hline
\end{tabular}

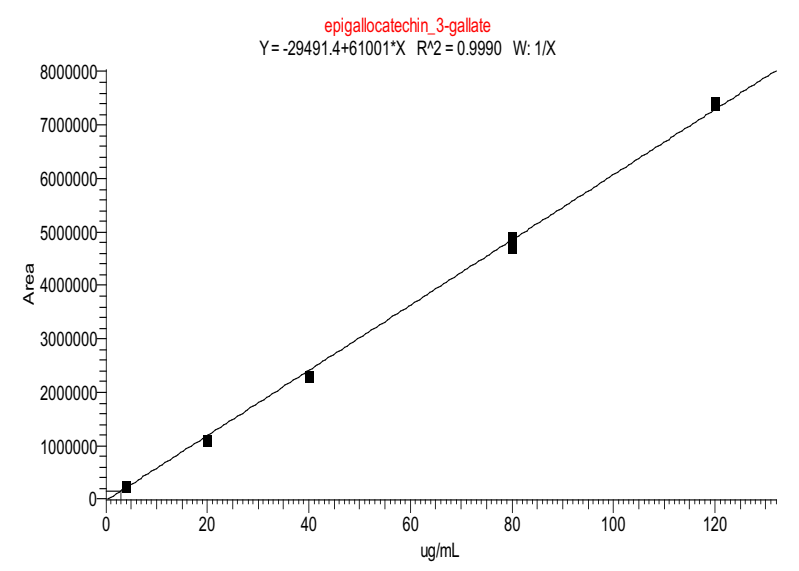

Figure 3.2.4. epigallokatechin-3-gallate Reaction Equation.

\subsubsection{Cafeic acid}

Cafeic acid values and reaction equation results are given in Table 3.2.5 and Fig. 3.2.5. Highest cafeic acid values were evaluated from Siyah Gemre variety and for the other varieties values were determined less or not found (NF). Cafeic acid was determined in 4 variety according to results. These varieties were Siyah Gemre, Devegozü, Antep Büzgüiü and Senirkent Dimriti.

Table 3.2.5. Cafeic acid results according to variety.

\begin{tabular}{|c|c|c|}
\hline Ak Gemre $\mathrm{HCl}$ & NF & $\mu \mathrm{g} / \mathrm{mL}$ \\
\hline Ak Gemre $\mathrm{MeOH}$ & NF & $\mu \mathrm{g} / \mathrm{mL}$ \\
\hline Ak Dimrit $\mathrm{HCl}$ & $\mathrm{NF}$ & $\mu \mathrm{g} / \mathrm{mL}$ \\
\hline Ak Dimrit $\mathrm{MeOH}$ & NF & $\mu \mathrm{g} / \mathrm{mL}$ \\
\hline Antep Büzgülü $\mathrm{HCl}$ & $\mathrm{NF}$ & $\mu \mathrm{g} / \mathrm{mL}$ \\
\hline Antep Büzgülü $\mathrm{MeOH}$ & NF & $\mu \mathrm{g} / \mathrm{mL}$ \\
\hline Burdur Dimriti $\mathrm{HCl}$ & NF & $\mu \mathrm{g} / \mathrm{mL}$ \\
\hline Burdur Dimriti MeOH & NF & $\mu \mathrm{g} / \mathrm{mL}$ \\
\hline Devegözü $\mathrm{HCl}$ & $\mathrm{NF}$ & $\mu \mathrm{g} / \mathrm{mL}$ \\
\hline Devegözü MeOH & $\mathrm{NF}$ & $\mu \mathrm{g} / \mathrm{mL}$ \\
\hline Kuş Yüreği HCl & $\mathrm{NF}$ & $\mu \mathrm{g} / \mathrm{mL}$ \\
\hline Kis Yüreği $\mathrm{MeOH}$ & $\mathrm{NF}$ & $\mu \mathrm{g} / \mathrm{mL}$ \\
\hline Marzımat $\mathrm{HCl}$ & $\mathrm{NF}$ & $\mu \mathrm{g} / \mathrm{mL}$ \\
\hline Marzımat $\mathrm{MeOH}$ & NF & $\mu \mathrm{g} / \mathrm{mL}$ \\
\hline Ak Gemre $20 \mu \mathrm{g} / \mathrm{mL} \mathrm{HCl}$ & $\mathrm{NF}$ & $\mu \mathrm{g} / \mathrm{mL}$ \\
\hline Ak Gemre $20 \mu \mathrm{g} / \mathrm{mL} \mathrm{MeOH}$ & $\mathrm{NF}$ & $\mu \mathrm{g} / \mathrm{mL}$ \\
\hline Ak Dimrit $20 \mu \mathrm{g} / \mathrm{mL} \mathrm{HCl}$ & $\mathrm{NF}$ & $\mu \mathrm{g} / \mathrm{mL}$ \\
\hline Ak Dimrit $20 \mu \mathrm{g} / \mathrm{mL} \mathrm{MeOH}$ & $\mathrm{NF}$ & $\mu \mathrm{g} / \mathrm{mL}$ \\
\hline Antep Büzgülü $20 \mu \mathrm{g} / \mathrm{mL} \mathrm{HCl}$ & $\mathrm{NF}$ & $\mu \mathrm{g} / \mathrm{mL}$ \\
\hline Antep Büzgülü $20 \mu \mathrm{g} / \mathrm{mL} \mathrm{MeOH}$ & 12,513 & $\mu \mathrm{g} / \mathrm{mL}$ \\
\hline Burdur Dimriti $20 \mu \mathrm{g} / \mathrm{mL} \mathrm{HCl}$ & $\mathrm{NF}$ & $\mu \mathrm{g} / \mathrm{mL}$ \\
\hline Burdur Dimriti $20 \mu \mathrm{g} / \mathrm{mL} \mathrm{MeOH}$ & $\mathrm{NF}$ & $\mu \mathrm{g} / \mathrm{mL}$ \\
\hline Devegözü $20 \mu \mathrm{g} / \mathrm{mL} \mathrm{HCl}$ & $\mathrm{NF}$ & $\mu \mathrm{g} / \mathrm{mL}$ \\
\hline Devegözü $20 \mu \mathrm{g} / \mathrm{mL} \mathrm{MeOH}$ & 8,885 & $\mu \mathrm{g} / \mathrm{mL}$ \\
\hline Kuş Yureği $20 \mu \mathrm{g} / \mathrm{mL} \mathrm{HCl}$ & $\mathrm{NF}$ & $\mu \mathrm{g} / \mathrm{mL}$ \\
\hline Kuş Yüreği $20 \mu \mathrm{g} / \mathrm{mL} \mathrm{MeOH}$ & $\mathrm{NF}$ & $\mu \mathrm{g} / \mathrm{mL}$ \\
\hline Marzımat $20 \mu \mathrm{g} / \mathrm{mL} \mathrm{HCl}$ & $\mathrm{NF}$ & $\mu \mathrm{g} / \mathrm{mL}$ \\
\hline Marzımat $20 \mu \mathrm{g} / \mathrm{mL} \mathrm{MeOH}$ & $\mathrm{NF}$ & $\mu \mathrm{g} / \mathrm{mL}$ \\
\hline Şam Büzgülü $20 \mu \mathrm{g} / \mathrm{mL} \mathrm{HCl}$ & $\mathrm{NF}$ & $\mu \mathrm{g} / \mathrm{mL}$ \\
\hline Şam Büzgülü $20 \mu \mathrm{g} / \mathrm{mL} \mathrm{MeOH}$ & $\mathrm{NF}$ & $\mu \mathrm{g} / \mathrm{mL}$ \\
\hline Senirkent Dimriti $20 \mu \mathrm{g} / \mathrm{mL} \mathrm{HCl}$ & $\mathrm{NF}$ & $\mu \mathrm{g} / \mathrm{mL}$ \\
\hline Senirkent Dimriti $20 \mu \mathrm{g} / \mathrm{mL} \mathrm{MeOH}$ & 10,665 & $\mu \mathrm{g} / \mathrm{mL}$ \\
\hline Siyah Gemre $20 \mu \mathrm{g} / \mathrm{mL} \mathrm{HCl}$ & $\mathrm{NF}$ & $\mu \mathrm{g} / \mathrm{mL}$ \\
\hline Siyah Gemre $20 \mu \mathrm{g} / \mathrm{mL} \mathrm{MeOH}$ & 14,825 & $\mu \mathrm{g} / \mathrm{mL}$ \\
\hline Tilki Kuyruğu $20 \mu \mathrm{g} / \mathrm{mL} \mathrm{HCl}$ & $\mathrm{NF}$ & $\mu \mathrm{g} / \mathrm{mL}$ \\
\hline Tilki Kuyruğu $20 \mu \mathrm{g} / \mathrm{mL} \mathrm{MeOH}$ & $\mathrm{NF}$ & $\mu \mathrm{g} / \mathrm{mL}$ \\
\hline Şam Büzgülü $\mathrm{HCl}$ & NF & $\mu \mathrm{g} / \mathrm{mL}$ \\
\hline
\end{tabular}


Table 3.2.5. Continued.

\begin{tabular}{|l|c|c|}
\hline Şam Büzgülü MeOH & NF & $\mu \mathrm{g} / \mathrm{mL}$ \\
\hline Senirkent DimritiHCl & NF & $\mu \mathrm{g} / \mathrm{mL}$ \\
\hline Senirkent Dimriti MeOH & NF & $\mu \mathrm{g} / \mathrm{mL}$ \\
\hline Siyah Gemre HCl & NF & $\mu \mathrm{g} / \mathrm{mL}$ \\
\hline Siyah Gemre MeOH & NF & $\mu \mathrm{g} / \mathrm{mL}$ \\
\hline STD1:20ppm_ & NF & $\mu \mathrm{g} / \mathrm{mL}$ \\
\hline Tilki Kuyruğu HCl & NF & $\mu \mathrm{g} / \mathrm{mL}$ \\
\hline Tilki Kuyruğu MeOH & NF & $\mu \mathrm{g} / \mathrm{mL}$ \\
\hline
\end{tabular}

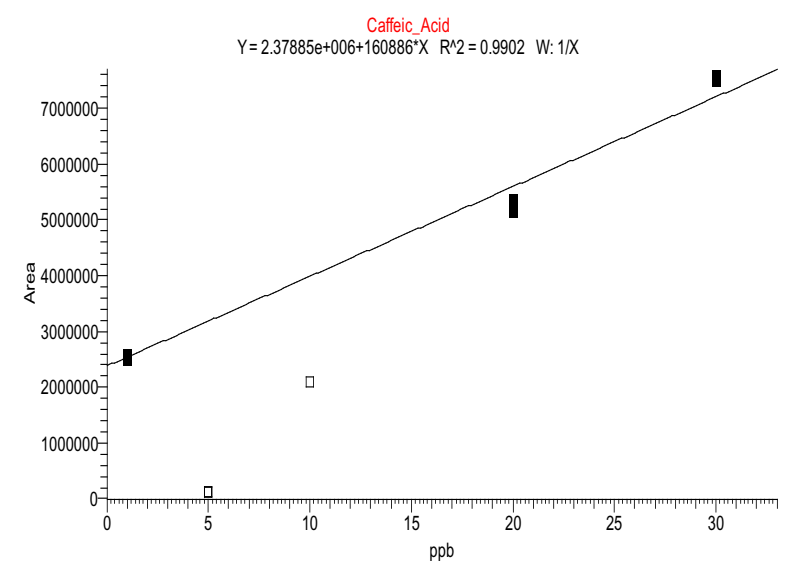

Figure 3.2.5. Cafeic acid reaction equation.

\subsubsection{Rutinhidrate}

Rutinhidrate values and reaction equation results are given in Table 3.2.6 and Fig. 3.2.6. Highest rutinhidrate values were evaluated from Marzımat and Antep Büzgülü varieites for the other varieties rutinhidrate values were determined less or not found (NF).

Table 3.2.6. Rutinhidrate results according to variety.

\begin{tabular}{|l|c|c|}
\hline Ak Gemre $\mathrm{HCl}$ & $\mathrm{NF}$ & $\mu \mathrm{g} / \mathrm{mL}$ \\
\hline Ak Gemre $\mathrm{MeOH}$ & $\mathrm{NF}$ & $\mu \mathrm{g} / \mathrm{mL}$ \\
\hline Ak Dimrit $\mathrm{HCl}$ & $\mathrm{NF}$ & $\mu \mathrm{g} / \mathrm{mL}$ \\
\hline Ak Dimrit $\mathrm{MeOH}$ & $\mathrm{NF}$ & $\mu \mathrm{g} / \mathrm{mL}$ \\
\hline Antep Büzgülü $\mathrm{HCl}$ & $\mathrm{NF}$ & $\mu \mathrm{g} / \mathrm{mL}$ \\
\hline Antep Büzgülü $\mathrm{MeOH}$ & $\mathrm{NF}$ & $\mu \mathrm{g} / \mathrm{mL}$ \\
\hline Burdur Dimriti $\mathrm{HCl}$ & $\mathrm{NF}$ & $\mu \mathrm{g} / \mathrm{mL}$ \\
\hline Burdur Dimriti $\mathrm{MeOH}$ & $\mathrm{NF}$ & $\mu \mathrm{g} / \mathrm{mL}$ \\
\hline Devegözü $\mathrm{HCl}$ & $\mathrm{NF}$ & $\mu \mathrm{g} / \mathrm{mL}$ \\
\hline Devegözü $\mathrm{MeOH}$ & $\mathrm{NF}$ & $\mu \mathrm{g} / \mathrm{mL}$ \\
\hline Kuş Yüreği $\mathrm{HCl}$ & $\mathrm{NF}$ & $\mu \mathrm{g} / \mathrm{mL}$ \\
\hline Kis Yüreği $\mathrm{MeOH}$ & $\mathrm{NF}$ & $\mu \mathrm{g} / \mathrm{mL}$ \\
\hline Marzımat $\mathrm{HCl}$ & $\mathrm{NF}$ & $\mu \mathrm{g} / \mathrm{mL}$ \\
\hline Marzımat $\mathrm{MeOH}$ & $\mathrm{NF}$ & $\mu \mathrm{g} / \mathrm{mL}$ \\
\hline
\end{tabular}

\begin{tabular}{|c|c|c|}
\hline Ak Gemre $20 \mu \mathrm{g} / \mathrm{mL} \mathrm{HCl}$ & $\mathrm{NF}$ & $\mu \mathrm{g} / \mathrm{mL}$ \\
\hline Ak Gemre $20 \mu \mathrm{g} / \mathrm{mL} \mathrm{MeOH}$ & 17,813 & $\mu \mathrm{g} / \mathrm{mL}$ \\
\hline Ak Dimrit $20 \mu \mathrm{g} / \mathrm{mL} \mathrm{HCl}$ & $\mathrm{NF}$ & $\mu \mathrm{g} / \mathrm{mL}$ \\
\hline Ak Dimrit $20 \mu \mathrm{g} / \mathrm{mL} \mathrm{MeOH}$ & $\mathrm{NF}$ & $\mu \mathrm{g} / \mathrm{mL}$ \\
\hline Antep Büzgülü $20 \mu \mathrm{g} / \mathrm{mL} \mathrm{HCl}$ & $\mathrm{NF}$ & $\mu \mathrm{g} / \mathrm{mL}$ \\
\hline Antep Büzgülü $20 \mu \mathrm{g} / \mathrm{mL} \mathrm{MeOH}$ & 25,099 & $\mu \mathrm{g} / \mathrm{mL}$ \\
\hline Burdur Dimriti $20 \mu \mathrm{g} / \mathrm{mL} \mathrm{HCl}$ & $\mathrm{NF}$ & $\mu \mathrm{g} / \mathrm{mL}$ \\
\hline Burdur Dimriti $20 \mu \mathrm{g} / \mathrm{mL} \mathrm{MeOH}$ & 23,745 & $\mu \mathrm{g} / \mathrm{mL}$ \\
\hline Devegözü $20 \mu \mathrm{g} / \mathrm{mL} \mathrm{HCl}$ & $\mathrm{NF}$ & $\mu \mathrm{g} / \mathrm{mL}$ \\
\hline Devegözü $20 \mu \mathrm{g} / \mathrm{mL} \mathrm{MeOH}$ & 21,233 & $\mu \mathrm{g} / \mathrm{mL}$ \\
\hline Kuş Yureği $20 \mu \mathrm{g} / \mathrm{mL} \mathrm{HCl}$ & $\mathrm{NF}$ & $\mu \mathrm{g} / \mathrm{mL}$ \\
\hline Kuş Yüreği $20 \mu \mathrm{g} / \mathrm{mL} \mathrm{MeOH}$ & 24,647 & $\mu \mathrm{g} / \mathrm{mL}$ \\
\hline Marzımat $20 \mu \mathrm{g} / \mathrm{mL} \mathrm{HCl}$ & 0,661 & $\mu \mathrm{g} / \mathrm{mL}$ \\
\hline Marzimat $20 \mu \mathrm{g} / \mathrm{mL} \mathrm{MeOH}$ & 25,987 & $\mu \mathrm{g} / \mathrm{mL}$ \\
\hline Şam Büzgülü $20 \mu \mathrm{g} / \mathrm{mL} \mathrm{HCl}$ & $\mathrm{NF}$ & $\mu \mathrm{g} / \mathrm{mL}$ \\
\hline Şam Büzgülü $20 \mu \mathrm{g} / \mathrm{mL} \mathrm{MeOH}$ & 21,719 & $\mu \mathrm{g} / \mathrm{mL}$ \\
\hline Senirkent Dimriti $20 \mu \mathrm{g} / \mathrm{mL} \mathrm{HCl}$ & $\mathrm{NF}$ & $\mu \mathrm{g} / \mathrm{mL}$ \\
\hline Senirkent Dimriti $20 \mu \mathrm{g} / \mathrm{mL} \mathrm{MeOH}$ & 20,070 & $\mu \mathrm{g} / \mathrm{mL}$ \\
\hline Siyah Gemre $20 \mu \mathrm{g} / \mathrm{mL} \mathrm{HCl}$ & $\mathrm{NF}$ & $\mu \mathrm{g} / \mathrm{mL}$ \\
\hline Siyah Gemre $20 \mu \mathrm{g} / \mathrm{mL} \mathrm{MeOH}$ & 20,665 & $\mu \mathrm{g} / \mathrm{mL}$ \\
\hline Tilki Kuyruğu $20 \mu \mathrm{g} / \mathrm{mL} \mathrm{HCl}$ & $\mathrm{NF}$ & $\mu \mathrm{g} / \mathrm{mL}$ \\
\hline Tilki Kuyruğu $20 \mu \mathrm{g} / \mathrm{mL} \mathrm{MeOH}$ & 21,066 & $\mu \mathrm{g} / \mathrm{mL}$ \\
\hline Şam Büzgülü HCl & NF & $\mu \mathrm{g} / \mathrm{mL}$ \\
\hline Şam Büzgülü MeOH & $\mathrm{NF}$ & $\mu \mathrm{g} / \mathrm{mL}$ \\
\hline Senirkent DimritiHCl & NF & $\mu \mathrm{g} / \mathrm{mL}$ \\
\hline Senirkent Dimriti MeOH & $\mathrm{NF}$ & $\mu \mathrm{g} / \mathrm{mL}$ \\
\hline Siyah Gemre $\mathrm{HCl}$ & NF & $\mu \mathrm{g} / \mathrm{mL}$ \\
\hline Siyah Gemre MeOH & $\mathrm{NF}$ & $\mu \mathrm{g} / \mathrm{mL}$ \\
\hline STD1:20ppm_ & 24,394 & $\mu \mathrm{g} / \mathrm{mL}$ \\
\hline Tilki Kuyruğu HCl & $\mathrm{NF}$ & $\mu \mathrm{g} / \mathrm{mL}$ \\
\hline Tilki Kuyruğu MeOH & $\mathrm{NF}$ & $\mu \mathrm{g} / \mathrm{mL}$ \\
\hline
\end{tabular}

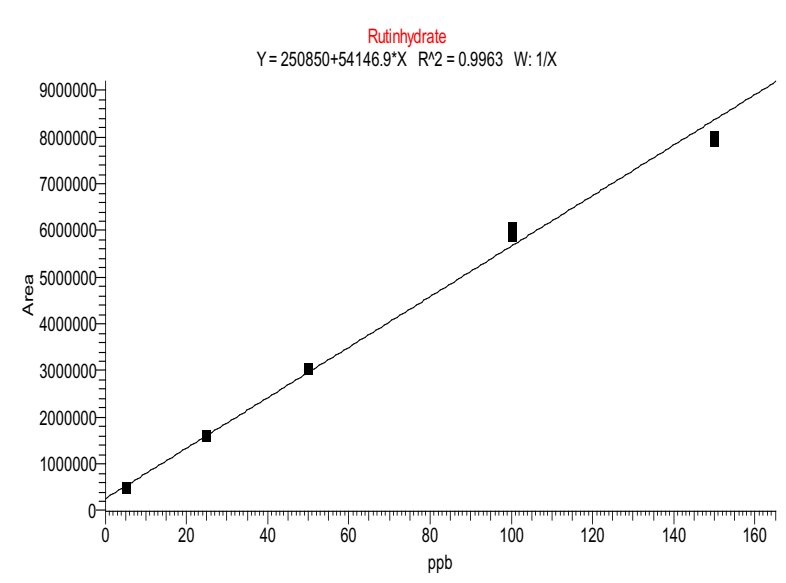

Figure 3.2.6. Rutinhidrate reaction equation. 


\subsection{7. p-Cumaric acid}

$\mathrm{p}$-Cumaric acid values and reaction equation results are given in Table 3.2.7 and Fig. 3.2.7. Highest p-cumaric acid values were evaluated from Burdur Dimriti and Kuş Yuregi varieties and for the other varieties values were determined less or not found (NF).

Table 3.2.7. $\mathrm{p}$-kumaric results according to variety.

\begin{tabular}{|c|c|c|}
\hline Ak Gemre $\mathrm{HCl}$ & NF & $\mu \mathrm{g} / \mathrm{mL}$ \\
\hline Ak Gemre $\mathrm{MeOH}$ & $\mathrm{NF}$ & $\mu \mathrm{g} / \mathrm{mL}$ \\
\hline Ak Dimrit $\mathrm{HCl}$ & NF & $\mu \mathrm{g} / \mathrm{mL}$ \\
\hline Ak Dimrit $\mathrm{MeOH}$ & $\mathrm{NF}$ & $\mu \mathrm{g} / \mathrm{mL}$ \\
\hline Antep Büzgülü $\mathrm{HCl}$ & NF & $\mu \mathrm{g} / \mathrm{mL}$ \\
\hline Antep Büzgülü $\mathrm{MeOH}$ & NF & $\mu \mathrm{g} / \mathrm{mL}$ \\
\hline Burdur Dimriti $\mathrm{HCl}$ & $\mathrm{NF}$ & $\mu \mathrm{g} / \mathrm{mL}$ \\
\hline Burdur Dimriti MeOH & $\mathrm{NF}$ & $\mu \mathrm{g} / \mathrm{mL}$ \\
\hline Devegözü $\mathrm{HCl}$ & NF & $\mu \mathrm{g} / \mathrm{mL}$ \\
\hline Devegözü MeOH & $\mathrm{NF}$ & $\mu \mathrm{g} / \mathrm{mL}$ \\
\hline Kuş Yüreği HCl & $\mathrm{NF}$ & $\mu \mathrm{g} / \mathrm{mL}$ \\
\hline Kus Yüreği MeOH & NF & $\mu \mathrm{g} / \mathrm{mL}$ \\
\hline Marzımat $\mathrm{HCl}$ & $\mathrm{NF}$ & $\mu \mathrm{g} / \mathrm{mL}$ \\
\hline Marzımat $\mathrm{MeOH}$ & NF & $\mu \mathrm{g} / \mathrm{mL}$ \\
\hline Ak Gemre $20 \mu \mathrm{g} / \mathrm{mL} \mathrm{HCl}$ & 0,255 & $\mu \mathrm{g} / \mathrm{mL}$ \\
\hline Ak Gemre $20 \mu \mathrm{g} / \mathrm{mL} \mathrm{MeOH}$ & 15,296 & $\mu \mathrm{g} / \mathrm{mL}$ \\
\hline Ak Dimrit $20 \mu \mathrm{g} / \mathrm{mL} \mathrm{HCl}$ & 0,935 & $\mu \mathrm{g} / \mathrm{mL}$ \\
\hline Ak Dimrit $20 \mu \mathrm{g} / \mathrm{mL} \mathrm{MeOH}$ & 15,760 & $\mu \mathrm{g} / \mathrm{mL}$ \\
\hline Antep Büzgülü $20 \mu \mathrm{g} / \mathrm{mL} \mathrm{HCl}$ & $\mathrm{NF}$ & $\mu \mathrm{g} / \mathrm{mL}$ \\
\hline Antep Büzgülü $20 \mu \mathrm{g} / \mathrm{mL} \mathrm{MeOH}$ & 15,993 & $\mu \mathrm{g} / \mathrm{mL}$ \\
\hline Burdur Dimriti $20 \mu \mathrm{g} / \mathrm{mL} \mathrm{HCl}$ & 0,613 & $\mu \mathrm{g} / \mathrm{mL}$ \\
\hline Burdur Dimriti $20 \mu \mathrm{g} / \mathrm{mL} \mathrm{MeOH}$ & 23,135 & $\mu \mathrm{g} / \mathrm{mL}$ \\
\hline Devegözü $20 \mu \mathrm{g} / \mathrm{mL} \mathrm{HCl}$ & 0,457 & $\mu \mathrm{g} / \mathrm{mL}$ \\
\hline Devegözü $20 \mu \mathrm{g} / \mathrm{mL} \mathrm{MeOH}$ & 18,154 & $\mu \mathrm{g} / \mathrm{mL}$ \\
\hline Kuş Yureği $20 \mu \mathrm{g} / \mathrm{mL} \mathrm{HCl}$ & 0,534 & $\mu \mathrm{g} / \mathrm{mL}$ \\
\hline Kuş Yüreği $20 \mu \mathrm{g} / \mathrm{mL} \mathrm{MeOH}$ & 24,102 & $\mu \mathrm{g} / \mathrm{mL}$ \\
\hline Marzımat $20 \mu \mathrm{g} / \mathrm{mL} \mathrm{HCl}$ & 1,033 & $\mu \mathrm{g} / \mathrm{mL}$ \\
\hline Marzımat $20 \mu \mathrm{g} / \mathrm{mL} \mathrm{MeOH}$ & 21,436 & $\mu \mathrm{g} / \mathrm{mL}$ \\
\hline Şam Büzgülü $20 \mu \mathrm{g} / \mathrm{mL} \mathrm{HCl}$ & 0,526 & $\mu \mathrm{g} / \mathrm{mL}$ \\
\hline Şam Büzgülü $20 \mu \mathrm{g} / \mathrm{mL} \mathrm{MeOH}$ & 17,821 & $\mu \mathrm{g} / \mathrm{mL}$ \\
\hline Senirkent Dimriti $20 \mu \mathrm{g} / \mathrm{mL} \mathrm{HCl}$ & 1,176 & $\mu \mathrm{g} / \mathrm{mL}$ \\
\hline Senirkent Dimriti $20 \mu \mathrm{g} / \mathrm{mL} \mathrm{MeOH}$ & 13,303 & $\mu \mathrm{g} / \mathrm{mL}$ \\
\hline Siyah Gemre $20 \mu \mathrm{g} / \mathrm{mL} \mathrm{HCl}$ & 1,125 & $\mu \mathrm{g} / \mathrm{mL}$ \\
\hline Siyah Gemre $20 \mu \mathrm{g} / \mathrm{mL} \mathrm{MeOH}$ & 18,029 & $\mu \mathrm{g} / \mathrm{mL}$ \\
\hline Tilki Kuyruğu $20 \mu \mathrm{g} / \mathrm{mL} \mathrm{HCl}$ & 0,443 & $\mu \mathrm{g} / \mathrm{mL}$ \\
\hline Tilki Kuyruğu 20 $\mu \mathrm{g} / \mathrm{mL} \mathrm{MeOH}$ & 14,035 & $\mu \mathrm{g} / \mathrm{mL}$ \\
\hline Şam Büzgülü $\mathrm{HCl}$ & NF & $\mu \mathrm{g} / \mathrm{mL}$ \\
\hline Şam Büzgülü MeOH & $\mathrm{NF}$ & $\mu \mathrm{g} / \mathrm{mL}$ \\
\hline Senirkent DimritiHCl & NF & $\mu \mathrm{g} / \mathrm{mL}$ \\
\hline
\end{tabular}

\begin{tabular}{|l|l|l|}
\hline Senirkent Dimriti $\mathrm{MeOH}$ & NF & $\mu \mathrm{g} / \mathrm{mL}$ \\
\hline Siyah Gemre $\mathrm{HCl}$ & NF & $\mu \mathrm{g} / \mathrm{mL}$ \\
\hline Siyah Gemre $\mathrm{MeOH}$ & NF & $\mu \mathrm{g} / \mathrm{mL}$ \\
\hline STD1:20ppm__ & 24,433 & $\mu \mathrm{g} / \mathrm{mL}$ \\
\hline Tilki Kuyruğu $\mathrm{HCl}$ & NF & $\mu \mathrm{g} / \mathrm{mL}$ \\
\hline Tilki Kuyruğu $\mathrm{MeOH}$ & NF & $\mu \mathrm{g} / \mathrm{mL}$ \\
\hline
\end{tabular}

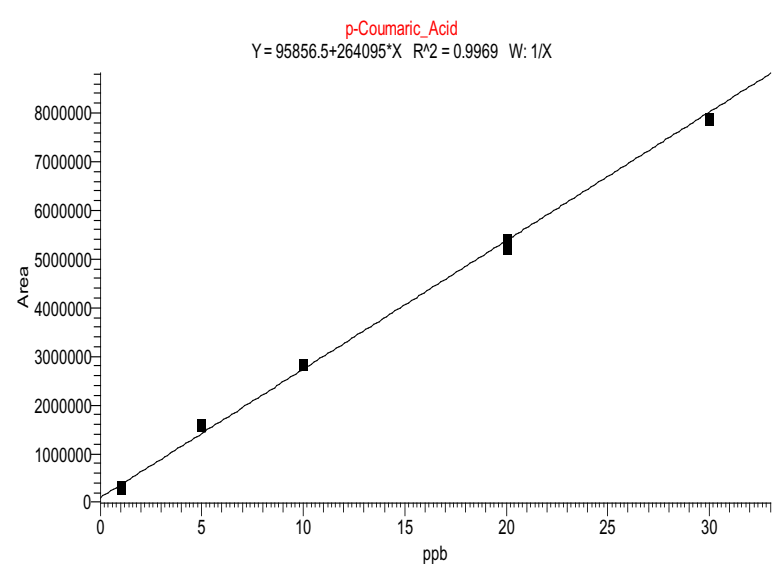

Figure 3.2. p-cumaric acid reaction equation.

\subsubsection{Ferulic acid}

Ferulic acid values and reaction equation results are given in Table 3.2.8 and Fig. 3.2.8. Highest ferulic acid values were evaluated from Kuş Yuregi, Burdur Dimriti and Marzımat varieties for the other varieties values were determined less or not found (NF).

Table 3.2.8. Ferulic acid results according to variety.

\begin{tabular}{|l|l|l|}
\hline Ak Gemre $\mathrm{HCl}$ & NF & $\mu \mathrm{g} / \mathrm{mL}$ \\
\hline Ak Gemre $\mathrm{MeOH}$ & NF & $\mu \mathrm{g} / \mathrm{mL}$ \\
\hline Ak Dimrit $\mathrm{HCl}$ & $\mathrm{NF}$ & $\mu \mathrm{g} / \mathrm{mL}$ \\
\hline Ak Dimrit $\mathrm{MeOH}$ & $\mathrm{NF}$ & $\mu \mathrm{g} / \mathrm{mL}$ \\
\hline Antep Büzgülü $\mathrm{HCl}$ & $\mathrm{NF}$ & $\mu \mathrm{g} / \mathrm{mL}$ \\
\hline Antep Büzgülü $\mathrm{MeOH}$ & $\mathrm{NF}$ & $\mu \mathrm{g} / \mathrm{mL}$ \\
\hline Burdur Dimriti $\mathrm{HCl}$ & $\mathrm{NF}$ & $\mu \mathrm{g} / \mathrm{mL}$ \\
\hline Burdur Dimriti $\mathrm{MeOH}$ & $\mathrm{NF}$ & $\mu \mathrm{g} / \mathrm{mL}$ \\
\hline Devegözü $\mathrm{HCl}$ & $\mathrm{NF}$ & $\mu \mathrm{g} / \mathrm{mL}$ \\
\hline Devegözü $\mathrm{MeOH}$ & $\mathrm{NF}$ & $\mu \mathrm{g} / \mathrm{mL}$ \\
\hline Kuş Yüreği $\mathrm{HCl}$ & $\mathrm{NF}$ & $\mu \mathrm{g} / \mathrm{mL}$ \\
\hline Kus Yüreği $\mathrm{MeOH}$ & 0,304 & $\mu \mathrm{g} / \mathrm{mL}$ \\
\hline Marzımat $\mathrm{HCl}$ & $\mathrm{NF}$ & $\mu \mathrm{g} / \mathrm{mL}$ \\
\hline Marzımat $\mathrm{MeOH}$ & 0,163 & $\mu \mathrm{g} / \mathrm{mL}$ \\
\hline Ak Gemre $20 \mu \mathrm{g} / \mathrm{mL} \mathrm{HCl}$ & 0,487 & $\mu \mathrm{g} / \mathrm{mL}$ \\
\hline Ak Gemre $20 \mu \mathrm{g} / \mathrm{mL} \mathrm{MeOH}$ & 19,012 & $\mu \mathrm{g} / \mathrm{mL}$ \\
\hline Ak Dimrit $20 \mu \mathrm{g} / \mathrm{mL} \mathrm{HCl}$ & 0,815 & $\mu \mathrm{g} / \mathrm{mL}$ \\
\hline Ak Dimrit $20 \mu \mathrm{g} / \mathrm{mL} \mathrm{MeOH}$ & 19,160 & $\mu \mathrm{g} / \mathrm{mL}$ \\
\hline
\end{tabular}


Table 3.2.8. Continued.

\begin{tabular}{|c|c|c|}
\hline Antep Büzgülü $20 \mu \mathrm{g} / \mathrm{mL} \mathrm{HCl}$ & 1,123 & $\mu \mathrm{g} / \mathrm{mL}$ \\
\hline Antep Büzgülü $20 \mu \mathrm{g} / \mathrm{mL} \mathrm{MeOH}$ & 19,305 & $\mu \mathrm{g} / \mathrm{mL}$ \\
\hline Burdur Dimriti $20 \mu \mathrm{g} / \mathrm{mL} \mathrm{HCl}$ & 0,738 & $\mu \mathrm{g} / \mathrm{mL}$ \\
\hline Burdur Dimriti $20 \mu \mathrm{g} / \mathrm{mL} \mathrm{MeOH}$ & 20,473 & $\mu \mathrm{g} / \mathrm{mL}$ \\
\hline Devegözü $20 \mu \mathrm{g} / \mathrm{mL} \mathrm{HCl}$ & 0,645 & $\mu \mathrm{g} / \mathrm{mL}$ \\
\hline Devegözü $20 \mu \mathrm{g} / \mathrm{mL} \mathrm{MeOH}$ & 21,802 & $\mu \mathrm{g} / \mathrm{mL}$ \\
\hline Kuş Yureği $20 \mu \mathrm{g} / \mathrm{mL} \mathrm{HCl}$ & 0,812 & $\mu \mathrm{g} / \mathrm{mL}$ \\
\hline Kuş Yüreği $20 \mu \mathrm{g} / \mathrm{mL} \mathrm{MeOH}$ & 21,300 & $\mu \mathrm{g} / \mathrm{mL}$ \\
\hline Marzımat $20 \mu \mathrm{g} / \mathrm{mL} \mathrm{HCl}$ & 1,117 & $\mu \mathrm{g} / \mathrm{mL}$ \\
\hline Marzımat $20 \mu \mathrm{g} / \mathrm{mL} \mathrm{MeOH}$ & 20,426 & $\mu \mathrm{g} / \mathrm{mL}$ \\
\hline Şam Büzgülü $20 \mu \mathrm{g} / \mathrm{mL} \mathrm{HCl}$ & 1,175 & $\mu \mathrm{g} / \mathrm{mL}$ \\
\hline Şam Büzgülü $20 \mu \mathrm{g} / \mathrm{mL} \mathrm{MeOH}$ & 21,628 & $\mu \mathrm{g} / \mathrm{mL}$ \\
\hline Senirkent Dimriti $20 \mu \mathrm{g} / \mathrm{mL} \mathrm{HCl}$ & 1,190 & $\mu \mathrm{g} / \mathrm{mL}$ \\
\hline Senirkent Dimriti $20 \mu \mathrm{g} / \mathrm{mL} \mathrm{MeOH}$ & 17,489 & $\mu \mathrm{g} / \mathrm{mL}$ \\
\hline Siyah Gemre $20 \mu \mathrm{g} / \mathrm{mL} \mathrm{HCl}$ & 1,108 & $\mu \mathrm{g} / \mathrm{mL}$ \\
\hline Siyah Gemre $20 \mu \mathrm{g} / \mathrm{mL} \mathrm{MeOH}$ & 21,635 & $\mu \mathrm{g} / \mathrm{mL}$ \\
\hline Tilki Kuyruğu $20 \mu \mathrm{g} / \mathrm{mL} \mathrm{HCl}$ & 0,730 & $\mu \mathrm{g} / \mathrm{mL}$ \\
\hline Tilki Kuyruğu $20 \mu \mathrm{g} / \mathrm{mL} \mathrm{MeOH}$ & 17,010 & $\mu \mathrm{g} / \mathrm{mL}$ \\
\hline Şam Büzgülü $\mathrm{HCl}$ & NF & $\mu \mathrm{g} / \mathrm{mL}$ \\
\hline Şam Büzgülü MeOH & NF & $\mu \mathrm{g} / \mathrm{mL}$ \\
\hline Senirkent DimritiHCl & $\mathrm{NF}$ & $\mu \mathrm{g} / \mathrm{mL}$ \\
\hline Senirkent Dimriti MeOH & $\mathrm{NF}$ & $\mu \mathrm{g} / \mathrm{mL}$ \\
\hline Siyah Gemre $\mathrm{HCl}$ & $\mathrm{NF}$ & $\mu \mathrm{g} / \mathrm{mL}$ \\
\hline Siyah Gemre MeOH & NF & $\mu \mathrm{g} / \mathrm{mL}$ \\
\hline STD1:20ppm_ & 21,275 & $\mu \mathrm{g} / \mathrm{mL}$ \\
\hline Tilki Kuyruğu HCl & NF & $\mu \mathrm{g} / \mathrm{mL}$ \\
\hline Tilki Kuyruğu MeOH & 0,222 & $\mu \mathrm{g} / \mathrm{mL}$ \\
\hline
\end{tabular}

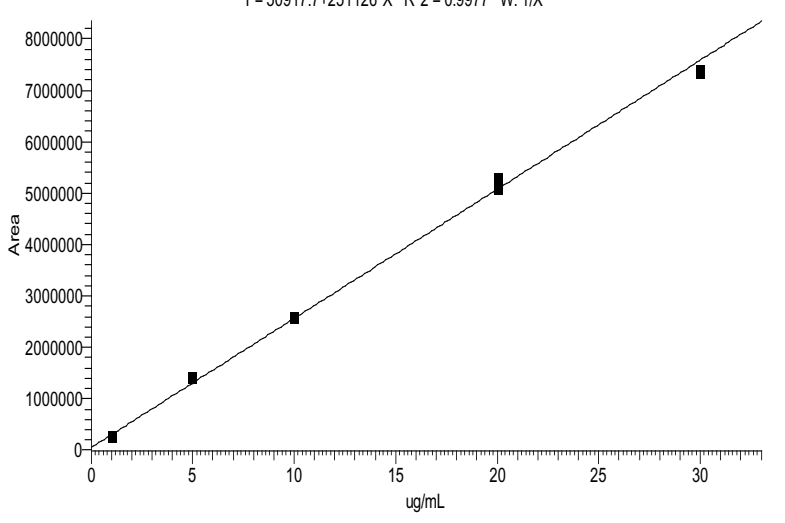

Figure 3.2.8. Ferulic acid reaction equation.

\subsubsection{Myricetin}

Myricetin values and reaction equation results are given in Table 3.2.9 and Fig. 3.2.9. Highest myricetin values were evaluated from Burdur Dimriti, Marzımat and Senirkent Dimriti for the other varieties values were determined less or not found (NF).
Table 3.2.9. Myricetin results according to variety.

\begin{tabular}{|c|c|c|}
\hline Ak Gemre $\mathrm{HCl}$ & NF & $\mu \mathrm{g} / \mathrm{mL}$ \\
\hline Ak Gemre $\mathrm{MeOH}$ & 0,700 & $\mu \mathrm{g} / \mathrm{mL}$ \\
\hline Ak Dimrit $\mathrm{HCl}$ & $\mathrm{NF}$ & $\mu \mathrm{g} / \mathrm{mL}$ \\
\hline Ak Dimrit $\mathrm{MeOH}$ & $\mathrm{NF}$ & $\mu \mathrm{g} / \mathrm{mL}$ \\
\hline Antep Büzgülü $\mathrm{HCl}$ & $\mathrm{NF}$ & $\mu \mathrm{g} / \mathrm{mL}$ \\
\hline Antep Büzgülü MeOH & $\mathrm{NF}$ & $\mu \mathrm{g} / \mathrm{mL}$ \\
\hline Burdur Dimriti $\mathrm{HCl}$ & $\mathrm{NF}$ & $\mu \mathrm{g} / \mathrm{mL}$ \\
\hline Burdur Dimriti $\mathrm{MeOH}$ & NF & $\mu \mathrm{g} / \mathrm{mL}$ \\
\hline Devegözü $\mathrm{HCl}$ & $\mathrm{NF}$ & $\mu \mathrm{g} / \mathrm{mL}$ \\
\hline Devegözü MeOH & NF & $\mu \mathrm{g} / \mathrm{mL}$ \\
\hline Kuş Yüreği $\mathrm{HCl}$ & NF & $\mu \mathrm{g} / \mathrm{mL}$ \\
\hline Kuş Yüreği MeOH & NF & $\mu \mathrm{g} / \mathrm{mL}$ \\
\hline Marzımat $\mathrm{HCl}$ & NF & $\mu \mathrm{g} / \mathrm{mL}$ \\
\hline Marzımat $\mathrm{MeOH}$ & $\mathrm{NF}$ & $\mu \mathrm{g} / \mathrm{mL}$ \\
\hline Ak Gemre $20 \mu \mathrm{g} / \mathrm{mL} \mathrm{HCl}$ & 15,571 & $\mu \mathrm{g} / \mathrm{mL}$ \\
\hline Ak Gemre $20 \mu \mathrm{g} / \mathrm{mL} \mathrm{MeOH}$ & 16,447 & $\mu \mathrm{g} / \mathrm{mL}$ \\
\hline Ak Dimrit $20 \mu \mathrm{g} / \mathrm{mL} \mathrm{HCl}$ & 19,916 & $\mu \mathrm{g} / \mathrm{mL}$ \\
\hline Ak Dimrit $20 \mu \mathrm{g} / \mathrm{mL} \mathrm{MeOH}$ & 17,005 & $\mu \mathrm{g} / \mathrm{mL}$ \\
\hline Antep Büzgülü $20 \mu \mathrm{g} / \mathrm{mL} \mathrm{HCl}$ & 2,110 & $\mu \mathrm{g} / \mathrm{mL}$ \\
\hline Antep Büzgülü $20 \mu \mathrm{g} / \mathrm{mL} \mathrm{MeOH}$ & 18,371 & $\mu \mathrm{g} / \mathrm{mL}$ \\
\hline Burdur Dimriti $20 \mu \mathrm{g} / \mathrm{mL} \mathrm{HCl}$ & 15,615 & $\mu \mathrm{g} / \mathrm{mL}$ \\
\hline Burdur Dimriti $20 \mu \mathrm{g} / \mathrm{mL} \mathrm{MeOH}$ & 21,625 & $\mu \mathrm{g} / \mathrm{mL}$ \\
\hline Devegözü $20 \mu \mathrm{g} / \mathrm{mL} \mathrm{HCl}$ & 8,522 & $\mu \mathrm{g} / \mathrm{mL}$ \\
\hline Devegözü $20 \mu \mathrm{g} / \mathrm{mL} \mathrm{MeOH}$ & 16,181 & $\mu \mathrm{g} / \mathrm{mL}$ \\
\hline Kuş Yureği $20 \mu \mathrm{g} / \mathrm{mL} \mathrm{HCl}$ & 17,383 & $\mu \mathrm{g} / \mathrm{mL}$ \\
\hline Kuş Yüreği $20 \mu \mathrm{g} / \mathrm{mL} \mathrm{MeOH}$ & 21,072 & $\mu \mathrm{g} / \mathrm{mL}$ \\
\hline Marzımat $20 \mu \mathrm{g} / \mathrm{mL} \mathrm{HCl}$ & 25,573 & $\mu \mathrm{g} / \mathrm{mL}$ \\
\hline Marzımat $20 \mu \mathrm{g} / \mathrm{mL} \mathrm{MeOH}$ & 20,914 & $\mu \mathrm{g} / \mathrm{mL}$ \\
\hline Şam Büzgülü $20 \mu \mathrm{g} / \mathrm{mL} \mathrm{HCl}$ & NF & $\mu \mathrm{g} / \mathrm{mL}$ \\
\hline Şam Büzgülü $20 \mu \mathrm{g} / \mathrm{mL} \mathrm{MeOH}$ & 14,014 & $\mu \mathrm{g} / \mathrm{mL}$ \\
\hline Senirkent Dimriti $20 \mu \mathrm{g} / \mathrm{mL} \mathrm{HCl}$ & $\mathrm{NF}$ & $\mu \mathrm{g} / \mathrm{mL}$ \\
\hline Senirkent Dimriti $20 \mu \mathrm{g} / \mathrm{mL} \mathrm{MeOH}$ & 16,534 & $\mu \mathrm{g} / \mathrm{mL}$ \\
\hline Siyah Gemre $20 \mu \mathrm{g} / \mathrm{mL} \mathrm{HCl}$ & 1,457 & $\mu \mathrm{g} / \mathrm{mL}$ \\
\hline Siyah Gemre $20 \mu \mathrm{g} / \mathrm{mL} \mathrm{MeOH}$ & 15,686 & $\mu \mathrm{g} / \mathrm{mL}$ \\
\hline Tilki Kuyruğu $20 \mu \mathrm{g} / \mathrm{mL} \mathrm{HCl}$ & 15,780 & $\mu \mathrm{g} / \mathrm{mL}$ \\
\hline 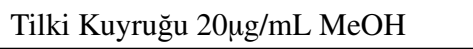 & 16,475 & $\mu \mathrm{g} / \mathrm{mL}$ \\
\hline Şam Büzgülü HCl & NF & $\mu \mathrm{g} / \mathrm{mL}$ \\
\hline Şam Büzgülü MeOH & $\mathrm{NF}$ & $\mu \mathrm{g} / \mathrm{mL}$ \\
\hline Senirkent DimritiHCl & NF & $\mu \mathrm{g} / \mathrm{mL}$ \\
\hline Senirkent Dimriti MeOH & $\mathrm{NF}$ & $\mu \mathrm{g} / \mathrm{mL}$ \\
\hline Siyah Gemre HCl & NF & $\mu \mathrm{g} / \mathrm{mL}$ \\
\hline Siyah Gemre MeOH & $\mathrm{NF}$ & $\mu \mathrm{g} / \mathrm{mL}$ \\
\hline STD1:20ppm_ & 18,060 & $\mu \mathrm{g} / \mathrm{mL}$ \\
\hline Tilki Kuyruğu HCl & NF & $\mu \mathrm{g} / \mathrm{mL}$ \\
\hline Tilki Kuyruğu MeOH & $\mathrm{NF}$ & $\mu \mathrm{g} / \mathrm{mL}$ \\
\hline
\end{tabular}




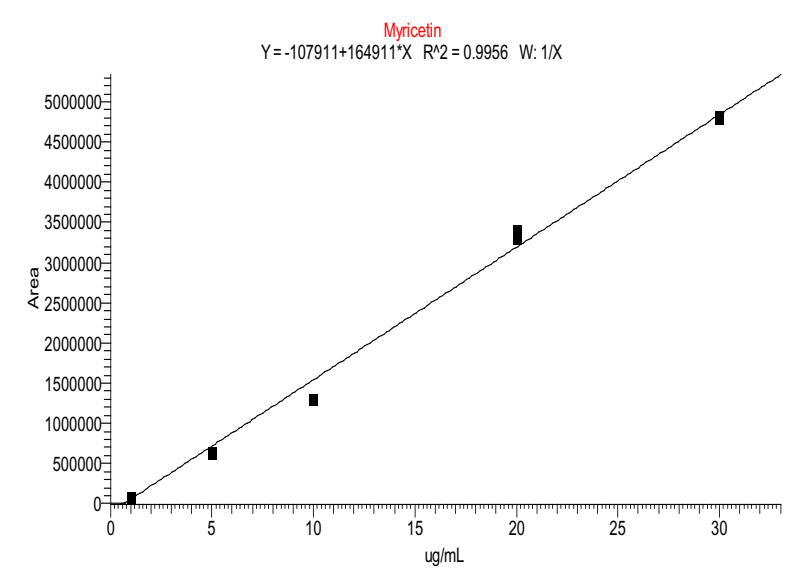

Figure 3.2.9. Myricetin reaction equation.

\subsubsection{Resveratrol}

Resveratrol values and reaction equation results are given in Table 3.2.10 and Fig. 3.2.10. Highest resveratrol values were evaluated from Burdur Dimriti, Kuş Yuregi, Sam Büzgülü, Siyah Gemre and Marzımat for the other varieties values were determined less or not found (NF).

Table 3.2.10. Resveratrol results according to variety.

\begin{tabular}{|c|c|c|}
\hline Ak Gemre $\mathrm{HCl}$ & NF & $\mu \mathrm{g} / \mathrm{mL}$ \\
\hline Ak Gemre $\mathrm{MeOH}$ & 0,010 & $\mu \mathrm{g} / \mathrm{mL}$ \\
\hline Ak Dimrit $\mathrm{HCl}$ & 0,540 & $\mu \mathrm{g} / \mathrm{mL}$ \\
\hline Ak Dimrit $\mathrm{MeOH}$ & 0,663 & $\mu \mathrm{g} / \mathrm{mL}$ \\
\hline Antep Büzgülü $\mathrm{HCl}$ & $\mathrm{NF}$ & $\mu \mathrm{g} / \mathrm{mL}$ \\
\hline Antep Büzgülü MeOH & NF & $\mu \mathrm{g} / \mathrm{mL}$ \\
\hline Burdur Dimriti $\mathrm{HCl}$ & NF & $\mu \mathrm{g} / \mathrm{mL}$ \\
\hline Burdur Dimriti $\mathrm{MeOH}$ & $\mathrm{NF}$ & $\mu \mathrm{g} / \mathrm{mL}$ \\
\hline Devegözü $\mathrm{HCl}$ & NF & $\mu \mathrm{g} / \mathrm{mL}$ \\
\hline Devegözü MeOH & 0,029 & $\mu \mathrm{g} / \mathrm{mL}$ \\
\hline Kuş Yüreği HCl & $\mathrm{NF}$ & $\mu \mathrm{g} / \mathrm{mL}$ \\
\hline Kus Yüreği MeOH & NF & $\mu \mathrm{g} / \mathrm{mL}$ \\
\hline Marzımat $\mathrm{HCl}$ & $\mathrm{NF}$ & $\mu \mathrm{g} / \mathrm{mL}$ \\
\hline Marzımat $\mathrm{MeOH}$ & 0,045 & $\mu \mathrm{g} / \mathrm{mL}$ \\
\hline Ak Gemre $20 \mu \mathrm{g} / \mathrm{mL} \mathrm{HCl}$ & $\mathrm{NF}$ & $\mu \mathrm{g} / \mathrm{mL}$ \\
\hline Ak Gemre $20 \mu \mathrm{g} / \mathrm{mL} \mathrm{MeOH}$ & NF & $\mu \mathrm{g} / \mathrm{mL}$ \\
\hline Ak Dimrit $20 \mu \mathrm{g} / \mathrm{mL} \mathrm{HCl}$ & 13,356 & $\mu \mathrm{g} / \mathrm{mL}$ \\
\hline Ak Dimrit $20 \mu \mathrm{g} / \mathrm{mL} \mathrm{MeOH}$ & 20,347 & $\mu \mathrm{g} / \mathrm{mL}$ \\
\hline Antep Büzgülü $20 \mu \mathrm{g} / \mathrm{mL} \mathrm{HCl}$ & 14,159 & $\mu \mathrm{g} / \mathrm{mL}$ \\
\hline Antep Büzgülü $20 \mu \mathrm{g} / \mathrm{mL} \mathrm{MeOH}$ & 21,958 & $\mu \mathrm{g} / \mathrm{mL}$ \\
\hline Burdur Dimriti $20 \mu \mathrm{g} / \mathrm{mL} \mathrm{HCl}$ & 12,321 & $\mu \mathrm{g} / \mathrm{mL}$ \\
\hline Burdur Dimriti $20 \mu \mathrm{g} / \mathrm{mL} \mathrm{MeOH}$ & 23,585 & $\mu \mathrm{g} / \mathrm{mL}$ \\
\hline
\end{tabular}

\begin{tabular}{|c|c|c|}
\hline Devegözü $20 \mu \mathrm{g} / \mathrm{mL} \mathrm{HCl}$ & 21,526 & $\mu \mathrm{g} / \mathrm{mL}$ \\
\hline Devegözü $20 \mu \mathrm{g} / \mathrm{mL} \mathrm{MeOH}$ & 20,555 & $\mu \mathrm{g} / \mathrm{mL}$ \\
\hline Kuş Yureği $20 \mu \mathrm{g} / \mathrm{mL} \mathrm{HCl}$ & 13,276 & $\mu \mathrm{g} / \mathrm{mL}$ \\
\hline Kuş Yüreği $20 \mu \mathrm{g} / \mathrm{mL} \mathrm{MeOH}$ & 25,125 & $\mu \mathrm{g} / \mathrm{mL}$ \\
\hline Marzımat $20 \mu \mathrm{g} / \mathrm{mL} \mathrm{HCl}$ & 27,981 & $\mu \mathrm{g} / \mathrm{mL}$ \\
\hline Marzımat $20 \mu \mathrm{g} / \mathrm{mL} \mathrm{MeOH}$ & 23,763 & $\mu \mathrm{g} / \mathrm{mL}$ \\
\hline Şam Büzgülü $20 \mu \mathrm{g} / \mathrm{mL} \mathrm{HCl}$ & 33,277 & $\mu \mathrm{g} / \mathrm{mL}$ \\
\hline Şam Büzgülü $20 \mu \mathrm{g} / \mathrm{mL} \mathrm{MeOH}$ & 22,074 & $\mu \mathrm{g} / \mathrm{mL}$ \\
\hline Senirkent Dimriti $20 \mu \mathrm{g} / \mathrm{mL} \mathrm{HCl}$ & 25,836 & $\mu \mathrm{g} / \mathrm{mL}$ \\
\hline Senirkent Dimriti $20 \mu \mathrm{g} / \mathrm{mL} \mathrm{MeOH}$ & 22,215 & $\mu \mathrm{g} / \mathrm{mL}$ \\
\hline Siyah Gemre $20 \mu \mathrm{g} / \mathrm{mL} \mathrm{HCl}$ & 11,021 & $\mu \mathrm{g} / \mathrm{mL}$ \\
\hline Siyah Gemre $20 \mu \mathrm{g} / \mathrm{mL} \mathrm{MeOH}$ & 22,248 & $\mu \mathrm{g} / \mathrm{mL}$ \\
\hline Tilki Kuyruğu $20 \mu \mathrm{g} / \mathrm{mL} \mathrm{HCl}$ & 13,540 & $\mu \mathrm{g} / \mathrm{mL}$ \\
\hline 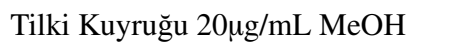 & 21,809 & $\mu \mathrm{g} / \mathrm{mL}$ \\
\hline Şam Büzgülü $\mathrm{HCl}$ & NF & $\mu \mathrm{g} / \mathrm{mL}$ \\
\hline Şam Büzgülü MeOH & 0,029 & $\mu \mathrm{g} / \mathrm{mL}$ \\
\hline Senirkent DimritiHCl & NF & $\mu \mathrm{g} / \mathrm{mL}$ \\
\hline Senirkent Dimriti MeOH & 0,014 & $\mu \mathrm{g} / \mathrm{mL}$ \\
\hline Siyah Gemre $\mathrm{HCl}$ & NF & $\mu \mathrm{g} / \mathrm{mL}$ \\
\hline Siyah Gemre $\mathrm{MeOH}$ & 0,016 & $\mu \mathrm{g} / \mathrm{mL}$ \\
\hline STD1:20ppm_ & 24,384 & $\mu \mathrm{g} / \mathrm{mL}$ \\
\hline Tilki Kuyruğu HCl & NF & $\mu \mathrm{g} / \mathrm{mL}$ \\
\hline Tilki Kuyruğu MeOH & NF & $\mu \mathrm{g} / \mathrm{mL}$ \\
\hline
\end{tabular}

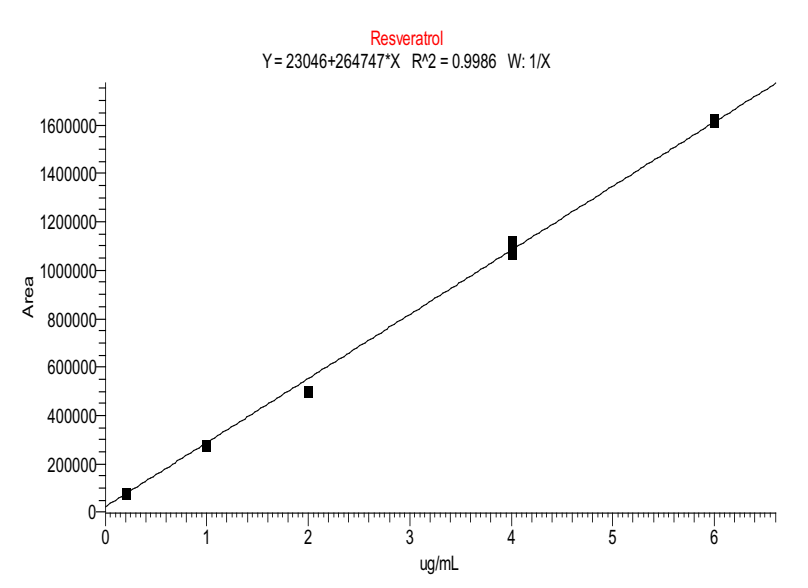

Figure 3.2.10. Resveratrol reaction equation.

\subsubsection{Quercetin}

Quercetin values and reaction equation results are given in Table 3.2.11 and Fig. 3.2.11. Highest quercetin values were evaluated from Burdur Dimriti, Kuş Yuregi and Sam Büzgülü varieties for the other varieties values were determined less or not found (NF). 
Table 3.2.11. Quercetin results according to variety.

\begin{tabular}{|c|c|c|}
\hline Ak Gemre $\mathrm{HCl}$ & $\mathrm{NF}$ & $\mu \mathrm{g} / \mathrm{mL}$ \\
\hline Ak Gemre $\mathrm{MeOH}$ & NF & $\mu \mathrm{g} / \mathrm{mL}$ \\
\hline Ak Dimrit $\mathrm{HCl}$ & NF & $\mu \mathrm{g} / \mathrm{mL}$ \\
\hline Ak Dimrit $\mathrm{MeOH}$ & NF & $\mu \mathrm{g} / \mathrm{mL}$ \\
\hline Antep Büzgülü $\mathrm{HCl}$ & 2,828 & $\mu \mathrm{g} / \mathrm{mL}$ \\
\hline Antep Büzgülü $\mathrm{MeOH}$ & 2,797 & $\mu \mathrm{g} / \mathrm{mL}$ \\
\hline Burdur Dimriti $\mathrm{HCl}$ & 2,827 & $\mu \mathrm{g} / \mathrm{mL}$ \\
\hline Burdur Dimriti MeOH & NF & $\mu \mathrm{g} / \mathrm{mL}$ \\
\hline Devegözü $\mathrm{HCl}$ & NF & $\mu \mathrm{g} / \mathrm{mL}$ \\
\hline Devegözü MeOH & NF & $\mu \mathrm{g} / \mathrm{mL}$ \\
\hline Kuş Yüreği HCl & NF & $\mu \mathrm{g} / \mathrm{mL}$ \\
\hline Kus Yüreği MeOH & NF & $\mu \mathrm{g} / \mathrm{mL}$ \\
\hline Marzımat $\mathrm{HCl}$ & 2,943 & $\mu \mathrm{g} / \mathrm{mL}$ \\
\hline Marzımat $\mathrm{MeOH}$ & NF & $\mu \mathrm{g} / \mathrm{mL}$ \\
\hline Ak Gemre $20 \mu \mathrm{g} / \mathrm{mL} \mathrm{HCl}$ & 22,233 & $\mu \mathrm{g} / \mathrm{mL}$ \\
\hline Ak Gemre $20 \mu \mathrm{g} / \mathrm{mL} \mathrm{MeOH}$ & 17,157 & $\mu \mathrm{g} / \mathrm{mL}$ \\
\hline Ak Dimrit $20 \mu \mathrm{g} / \mathrm{mL} \mathrm{HCl}$ & 27,403 & $\mu \mathrm{g} / \mathrm{mL}$ \\
\hline Ak Dimrit $20 \mu \mathrm{g} / \mathrm{mL} \mathrm{MeOH}$ & 16,543 & $\mu \mathrm{g} / \mathrm{mL}$ \\
\hline Antep Büzgülü $20 \mu \mathrm{g} / \mathrm{mL} \mathrm{HCl}$ & 22,530 & $\mu \mathrm{g} / \mathrm{mL}$ \\
\hline Antep Büzgülü $20 \mu \mathrm{g} / \mathrm{mL} \mathrm{MeOH}$ & 16,206 & $\mu \mathrm{g} / \mathrm{mL}$ \\
\hline Burdur Dimriti $20 \mu \mathrm{g} / \mathrm{mL} \mathrm{HCl}$ & 25,551 & $\mu \mathrm{g} / \mathrm{mL}$ \\
\hline Burdur Dimriti $20 \mu \mathrm{g} / \mathrm{mL} \mathrm{MeOH}$ & 16,087 & $\mu \mathrm{g} / \mathrm{mL}$ \\
\hline Devegözü $20 \mu \mathrm{g} / \mathrm{mL} \mathrm{HCl}$ & 23,352 & $\mu \mathrm{g} / \mathrm{mL}$ \\
\hline Devegözü $20 \mu \mathrm{g} / \mathrm{mL} \mathrm{MeOH}$ & 23,139 & $\mu \mathrm{g} / \mathrm{mL}$ \\
\hline Kuş Yureği $20 \mu \mathrm{g} / \mathrm{mL} \mathrm{HCl}$ & 32,322 & $\mu \mathrm{g} / \mathrm{mL}$ \\
\hline Kuş Yüreği $20 \mu \mathrm{g} / \mathrm{mL} \mathrm{MeOH}$ & 17,722 & $\mu \mathrm{g} / \mathrm{mL}$ \\
\hline Marzımat $20 \mu \mathrm{g} / \mathrm{mL} \mathrm{HCl}$ & 21,042 & $\mu \mathrm{g} / \mathrm{mL}$ \\
\hline Marzımat $20 \mu \mathrm{g} / \mathrm{mL} \mathrm{MeOH}$ & 12,886 & $\mu \mathrm{g} / \mathrm{mL}$ \\
\hline Şam Büzgülü $20 \mu \mathrm{g} / \mathrm{mL} \mathrm{HCl}$ & 12,757 & $\mu \mathrm{g} / \mathrm{mL}$ \\
\hline Şam Büzgülü $20 \mu \mathrm{g} / \mathrm{mL} \mathrm{MeOH}$ & 24,164 & $\mu \mathrm{g} / \mathrm{mL}$ \\
\hline Senirkent Dimriti $20 \mu \mathrm{g} / \mathrm{mL} \mathrm{HCl}$ & 17,112 & $\mu \mathrm{g} / \mathrm{mL}$ \\
\hline Senirkent Dimriti $20 \mu \mathrm{g} / \mathrm{mL} \mathrm{MeOH}$ & NF & $\mu \mathrm{g} / \mathrm{mL}$ \\
\hline Siyah Gemre $20 \mu \mathrm{g} / \mathrm{mL} \mathrm{HCl}$ & 2,798 & $\mu \mathrm{g} / \mathrm{mL}$ \\
\hline Siyah Gemre $20 \mu \mathrm{g} / \mathrm{mL} \mathrm{MeOH}$ & NF & $\mu \mathrm{g} / \mathrm{mL}$ \\
\hline 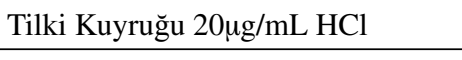 & $\mathrm{NF}$ & $\mu \mathrm{g} / \mathrm{mL}$ \\
\hline Tilki Kuyruğu $20 \mu \mathrm{g} / \mathrm{mL} \mathrm{MeOH}$ & NF & $\mu \mathrm{g} / \mathrm{mL}$ \\
\hline Şam Büzgülü HCl & NF & $\mu \mathrm{g} / \mathrm{mL}$ \\
\hline Şam Büzgülü $\mathrm{MeOH}$ & NF & $\mu \mathrm{g} / \mathrm{mL}$ \\
\hline Senirkent DimritiHCl & NF & $\mu \mathrm{g} / \mathrm{mL}$ \\
\hline Senirkent Dimriti MeOH & $\mathrm{NF}$ & $\mu \mathrm{g} / \mathrm{mL}$ \\
\hline Siyah Gemre HCl & NF & $\mu \mathrm{g} / \mathrm{mL}$ \\
\hline Siyah Gemre $\mathrm{MeOH}$ & NF & $\mu \mathrm{g} / \mathrm{mL}$ \\
\hline STD1:20ppm_ & 18,188 & $\mu \mathrm{g} / \mathrm{mL}$ \\
\hline Tilki Kuyruğu HCl & 2,997 & $\mu \mathrm{g} / \mathrm{mL}$ \\
\hline Tilki Kuyruğu MeOH & $\mathrm{NF}$ & $\mu \mathrm{g} / \mathrm{mL}$ \\
\hline
\end{tabular}

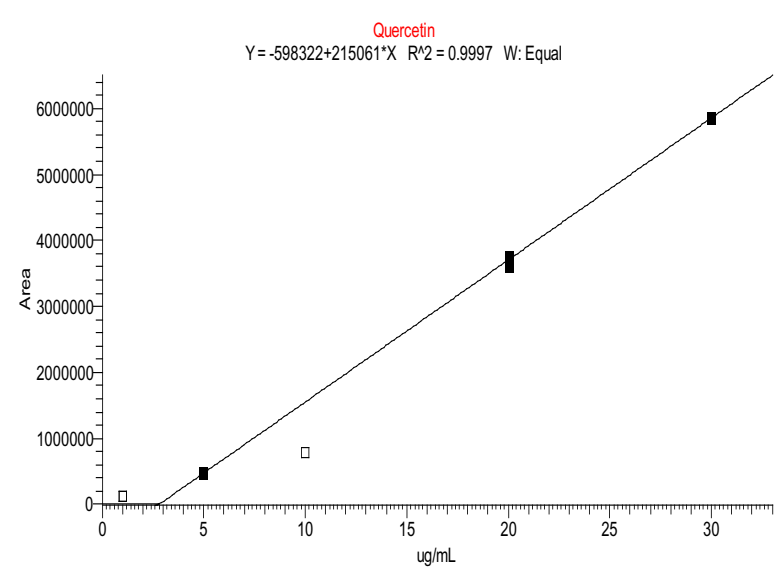

Figure 3.2.11. Quercetin acid reaction equation.

\subsubsection{Kaemferol}

Kaemfeol values and reaction equation results are given in Table 3.2.12 and Fig. 3.2.12 Highest kaemferol values were evaluated from Ak Dimrit, Burdur Dimriti, Kuş Yuregi and Marzımat and for the other varieties values were determined less or not found (NF).

Table 3.2.12. Kaemferol results according to variety.

\begin{tabular}{|l|l|l|}
\hline Ak Gemre $\mathrm{HCl}$ & $\mathrm{NF}$ & $\mu \mathrm{g} / \mathrm{mL}$ \\
\hline Ak Gemre MeOH & $\mathrm{NF}$ & $\mu \mathrm{g} / \mathrm{mL}$ \\
\hline Ak Dimrit $\mathrm{HCl}$ & $\mathrm{NF}$ & $\mu \mathrm{g} / \mathrm{mL}$ \\
\hline Ak Dimrit MeOH & $\mathrm{NF}$ & $\mu \mathrm{g} / \mathrm{mL}$ \\
\hline Antep Büzgülü $\mathrm{HCl}$ & $\mathrm{NF}$ & $\mu \mathrm{g} / \mathrm{mL}$ \\
\hline Antep Büzgülü MeOH & $\mathrm{NF}$ & $\mu \mathrm{g} / \mathrm{mL}$ \\
\hline Burdur Dimriti $\mathrm{HCl}$ & $\mathrm{NF}$ & $\mu \mathrm{g} / \mathrm{mL}$ \\
\hline Burdur Dimriti MeOH & $\mathrm{NF}$ & $\mu \mathrm{g} / \mathrm{mL}$ \\
\hline Devegözü $\mathrm{HCl}$ & $\mathrm{NF}$ & $\mu \mathrm{g} / \mathrm{mL}$ \\
\hline Devegözü MeOH & $\mathrm{NF}$ & $\mu \mathrm{g} / \mathrm{mL}$ \\
\hline Kuş Yüreği $\mathrm{HCl}$ & $\mathrm{NF}$ & $\mu \mathrm{g} / \mathrm{mL}$ \\
\hline Kuş Yüreği MeOH & $\mathrm{NF}$ & $\mu \mathrm{g} / \mathrm{mL}$ \\
\hline Marzımat $\mathrm{HCl}$ & $\mathrm{NF}$ & $\mu \mathrm{g} / \mathrm{mL}$ \\
\hline Marzımat MeOH & $\mathrm{NF}$ & $\mu \mathrm{g} / \mathrm{mL}$ \\
\hline Ak Gemre $20 \mu \mathrm{g} / \mathrm{mL} \mathrm{HCl}$ & 33,921 & $\mu \mathrm{g} / \mathrm{mL}$ \\
\hline Ak Gemre $20 \mu \mathrm{g} / \mathrm{mL} \mathrm{MeOH}$ & 21,516 & $\mu \mathrm{g} / \mathrm{mL}$ \\
\hline Ak Dimrit $20 \mu \mathrm{g} / \mathrm{mL} \mathrm{HCl}$ & 48,564 & $\mu \mathrm{g} / \mathrm{mL}$ \\
\hline Ak Dimrit $20 \mu \mathrm{g} / \mathrm{mL} \mathrm{MeOH}$ & 30,590 & $\mu \mathrm{g} / \mathrm{mL}$ \\
\hline Antep Büzgülü $20 \mu \mathrm{g} / \mathrm{mL} \mathrm{HCl}$ & 32,820 & $\mu \mathrm{g} / \mathrm{mL}$ \\
\hline Antep Büzgülü $20 \mu \mathrm{g} / \mathrm{mL} \mathrm{MeOH}$ & 25,742 & $\mu \mathrm{g} / \mathrm{mL}$ \\
\hline Burdur Dimriti $20 \mu \mathrm{g} / \mathrm{mL} \mathrm{HCl}$ & 33,253 & $\mu \mathrm{g} / \mathrm{mL}$ \\
\hline Burdur Dimriti $20 \mu \mathrm{g} / \mathrm{mL} \mathrm{MeOH}$ & 44,727 & $\mu \mathrm{g} / \mathrm{mL}$ \\
\hline Devegözü $20 \mu \mathrm{g} / \mathrm{mL} \mathrm{HCl}$ & 33,573 & $\mu \mathrm{g} / \mathrm{mL}$ \\
\hline Devegözü $20 \mu \mathrm{g} / \mathrm{mL} \mathrm{MeOH}$ & 26,654 & $\mu \mathrm{g} / \mathrm{mL}$ \\
\hline Kuş Yureği $20 \mu \mathrm{g} / \mathrm{mL} \mathrm{HCl}$ & 35,244 & $\mu \mathrm{g} / \mathrm{mL}$ \\
\hline
\end{tabular}


Table 3.2.12. Continued.

\begin{tabular}{|l|l|l|}
\hline Kuş Yüreği $20 \mu \mathrm{g} / \mathrm{mL} \mathrm{MeOH}$ & 44,143 & $\mu \mathrm{g} / \mathrm{mL}$ \\
\hline Marzımat $20 \mu \mathrm{g} / \mathrm{mL} \mathrm{HCl}$ & 59,177 & $\mu \mathrm{g} / \mathrm{mL}$ \\
\hline Marzımat $20 \mu \mathrm{g} / \mathrm{mL} \mathrm{MeOH}$ & 41,558 & $\mu \mathrm{g} / \mathrm{mL}$ \\
\hline Şam Büzgülü $20 \mu \mathrm{g} / \mathrm{mL} \mathrm{HCl}$ & 32,761 & $\mu \mathrm{g} / \mathrm{mL}$ \\
\hline Şam Büzgülü $20 \mu \mathrm{g} / \mathrm{mL} \mathrm{MeOH}$ & 22,796 & $\mu \mathrm{g} / \mathrm{mL}$ \\
\hline Senirkent Dimriti $20 \mu \mathrm{g} / \mathrm{mL} \mathrm{HCl}$ & 32,553 & $\mu \mathrm{g} / \mathrm{mL}$ \\
\hline Senirkent Dimriti $20 \mu \mathrm{g} / \mathrm{mL} \mathrm{MeOH}$ & 27,701 & $\mu \mathrm{g} / \mathrm{mL}$ \\
\hline Siyah Gemre $20 \mu \mathrm{g} / \mathrm{mL} \mathrm{HCl}$ & 32,158 & $\mu \mathrm{g} / \mathrm{mL}$ \\
\hline Siyah Gemre $20 \mu \mathrm{g} / \mathrm{mL} \mathrm{MeOH}$ & 25,292 & $\mu \mathrm{g} / \mathrm{mL}$ \\
\hline Tilki Kuyruğu $20 \mu \mathrm{g} / \mathrm{mL} \mathrm{HCl}$ & 33,137 & $\mu \mathrm{g} / \mathrm{mL}$ \\
\hline Tilki Kuyruğu $20 \mu \mathrm{g} / \mathrm{mL} \mathrm{MeOH}$ & 31,119 & $\mu \mathrm{g} / \mathrm{mL}$ \\
\hline Şam Büzgülü HCl & $\mathrm{NF}$ & $\mu \mathrm{g} / \mathrm{mL}$ \\
\hline Şam Büzgülü MeOH & $\mathrm{NF}$ & $\mu \mathrm{g} / \mathrm{mL}$ \\
\hline Senirkent DimritiHCl & $\mathrm{NF}$ & $\mu \mathrm{g} / \mathrm{mL}$ \\
\hline Senirkent Dimriti MeOH & $\mathrm{NF}$ & $\mu \mathrm{g} / \mathrm{mL}$ \\
\hline Siyah Gemre HCl & $\mathrm{NF}$ & $\mu \mathrm{g} / \mathrm{mL}$ \\
\hline Siyah Gemre MeOH & $\mathrm{NF}$ & $\mu \mathrm{g} / \mathrm{mL}$ \\
\hline STD1:20ppm_ & 37,396 & $\mu \mathrm{g} / \mathrm{mL}$ \\
\hline Tilki Kuyruğu HCl & $\mathrm{NF}$ & $\mu \mathrm{g} / \mathrm{mL}$ \\
\hline Tilki Kuyruğu MeOH & $\mathrm{NF}$ & $\mu \mathrm{g} / \mathrm{mL}$ \\
\hline
\end{tabular}

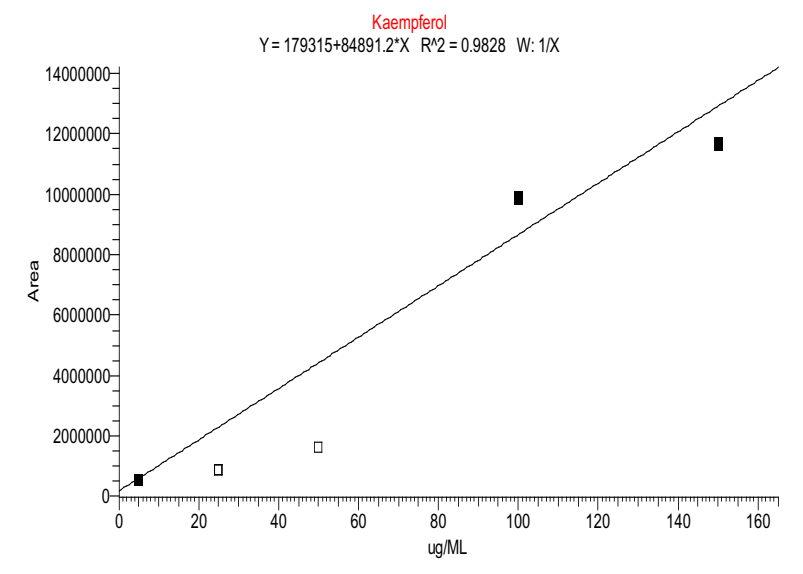

Figure 3.2.12. Kaenferol acid reaction equation.

\subsection{Conclusion}

When quality features were evaluated Burdur dimriti variety was determined good for table grape consumption and must production. It can be determined as an earlier variety for the region. Antep Büzgülü, Şam Büzgülü and Siyah Gemre varieties have homogen grape skin colour and good bunch and berry structures for table consumption. They were evaluated for table consumption for late time harvested varieties. Ak Gemre, Marzımat, Devegözü, Ak Dimrit, Senirkent Dimriti and Kuş Yüregi varieties were evaluated better for must production than table grape production. These varieties are advised to be used in must production. Ak Dimrit, Burdur Dimriti, Antep Büzgülü and Şam Büzgülü were evaluated good for yield.

11 local grape varieties were evaluated for 12 phenolic compounds (Gallic acid, cafeic acid, p-cumaric acid, ferulic acid, catechin, epicatechin, epicatechin gallate, epigallocatechin, epigallocatechin-3-gallate, kaemferol, myricetin, rutin hydrate, resveratrol and quercetin). Phenolic substances varied between 0 and $101.466 \mu \mathrm{g} / \mathrm{ml}$ in frozen berry samples varying to grape variety. When phenolic contents were compared other studies for local grape varieties, results of our study values were acceptable according to previous studies. Aras (2006), Orak (2007) and Şensoy (2012) had studied local grape cultivars phenolic contents in Turkey. We got nearly same results with their studies. Phenolic compounds were not evaluated significantly highest but some varieties were determined hopeful for some phenolics. Egigallocatechin-3-gallat resvreratrol, Kamefreol quersetin were determined significant level in some varieties. Epigallocatechin content was determined high in Senirkent Dimriti variety, epigallokatechin content determined high in Siyah Gemre variety. Rutinhdate was determined in all varieties except Ak Dimrit variety. Myricetin was determined high in Burdur Dimriti, Marzımat and Kuş Yuregi varieties. Resveratrol was determined high in Şam Büzgülü and antep Büzgülü varieties. Quercetin was determined high in Ak Dimrit, Kus Yuregi and Marzımat varieties. Antep Büzgülü, Burdur Dimriti, Kuş Yuregi, Senirkent Dimriti and Siyah Gemre varieties were determined hopeful varieties for future studies.

Researches on phenolic content of local grape varieties must be continued. Local grape varieties can be a good source of phenolics. Some local varieties cannot be good for table consumption, drying and other purposes but can be used for health because of their high phenolic contents. Studies and researches about local grape varieties phenolics will be continued and researches on hopeful varieties will be planned to be done in the future by adding different treatments like water stress, pruning, etc. Different treatments may result better phenolic contents in hopeful local grape varieties. Phenolics of local grape varieties will be continued by using this first research data.

\section{References}

[1] Puupponen-Pimiä R., Nohynek L., Meier C., Kähkönen M., Heinonen M., Hopia A. and OksmanCaldentey K.M., Antimicrobial properties of phenolic compounds from berries, Journal of Applied Microbiology 90, 2001, 494-507 pp.

[2] Hogan S., Zhang L., Chung H., Huang Z., Zoecklein B., Li J., et al., Antioxidant properties and bioactive components of Norton (Vitis aestivalis) and Cabernet Franc (Vitis vinifera) wine grapes, LWT-Food Science and Technology 42, 2009, 1269_ $1274 \mathrm{pp}$.

[3] Kähkönen M.P. and Heinonen M., Antioxidant activity of anthocyanins and their aglycons, Journal of Agricultural and Food Chemistry 51, 2003, 628-633 pp.

[4] Yilmaz Y.T. and Toledo R.T., Oxygen radical absorbance capacities of grape/wine industry byproducts and effect of solvent type on extraction of grape seed polyphenols, Journal of Food Composition and Analysis 19, 2006, 41-48. 
[5] Orak HH. Total antioxidant activities, phenolics, anthocyanins, polyphenoloxidase activities of selected red grape cultivars and their correlations. Sci Hortic. 2007; 111: 235-41 pp.

[6] Ivanova-Petropulos V, Ricci A, Nedelkovski D, Dimovska V, Parpinello GP, Versari A. Targeted analysis of bioactive phenolic compounds and antioxidant activity of Macedonian red wines. Food Chem. 2015; 171: 412-20 pp.

[7] Conde C, Silva P, Fontes N, Dias ACP, Tavares RM, Sousa M. J, et al. Biochemical changes throughout grape berry development and fruit and wine quality. Global Science Books, Food, 2007;1(1): 1-22 pp.

[8] Zerihun A, McClymont L, Lanyon D, Goodwin I, Gibberd M. Deconvoluting effects of vine and soil properties on grape berry composition. J Sci Food Agric. 2015; 95: 193-203pp.

[9] Pereira GE, Gaudillere J. P, Leeuwen CV, Hilbert G, Maucourt M, Deborde C, et al. ${ }^{1} \mathrm{H}$ NMR metabolite fingerprints of grape berry: comparison of vintage and soil effects in Bordeaux grapevine growing areas. Anal Chim Acta. 2006; 563 (1-2): 346-52 pp.

[10] Kafkas, E., A. Bozdoğan, A. Burgut, N. Türemiş, S. Paydaş Karg1, and T. Cabaroglu (2006). Total phenolic and anthocyanin contents in some berry fruit. II. Ulusal Üzümsü Meyveler Sempozyumu, Tokat, 309-312 pp. (in Turkish)
[11] Saldamli, I. (2007). Food Chemistry. Hacettepe Üniversitesi Yayınları. Ankara, 463-492. (In Turkish)

[12] Nizamlioglu, N. M. and S. Nas (2010). Phenolic compounds in fruit and vegetable and their importance. Gida Teknolojileri Elektronik Dergisi. 5(1): 20-35 pp. (in Turkish)

[13] Kedage, V.V., J.C. Tilak, G.B. Dixit, T.P.A. Devasagayam, and M. Mhatre (2007). A study of antioxidant properties of some varieties of grapes (Vitis vinifera L.). Critical Reviews in Food Science and Nutrition. 47(7): 175-185 pp.

[14] Negro, C., L. Tommasi, and A. Miceli (2003). Phenolic compounds and antioxidant activity from red grape marc extracts. Bioresource Technology 87: 41-44 pp.

[15] Cemeroglu, B., A. Yemenicioglu, and M. Ozkan (2004). Contents of Fruit and Vegetable. 1. Fruit and Vegetable Processing Technology (Editor: B. Cemeroglu). 2. Başkent Klişe Matbaacılık, 1. Ankara, 670 p., (in Turkish)

[16] Gargın, S., Göktas, A., Altındişli, A., 2010. Quality and Yield Performance of Popular Turkish Wine Varities (Kalecik Karasi and Narince) In Lake Region Ecological Conditions. 33rd World Congress of Vine and Wine 21-27.06.2010, Georgia.

[17] Şensoy, G.İ.R., 2012, Determination of phenolic substances and antioxidant activities in some grape cultivars by HPLC, The Journal of Animal and Plant Sciences, 22(2): 448-451 pp. 